\title{
Expert consensus statement
}

\section{Recommendations of the Spanish Societies of Radiation Oncology (SEOR), Nuclear Medicine \& Molecular Imaging (SEMNiM), and Medical Physics (SEFM) on ${ }^{18}$ F-FDG PET-CT for radiotherapy treatment planning}

\section{Begoña Caballero Perea ${ }^{a, *}$, Antonio Cabrera Villegas ${ }^{b}$, José Miguel Delgado Rodríguez ${ }^{c}$, María José García Velloso ${ }^{d}$, Ana María García Vicente ${ }^{e}$, Carlos Huerga Cabrerizof, Rosa Morera López ${ }^{g}$, Luis Alberto Pérez Romasanta ${ }^{h}$, Moisés Sáez Beltrán ${ }^{i}$, The Cooperative Group for PET-CT in Radiotherapy Treatment Planning ${ }^{1}$}

a Department of Radiation Oncology, Hospital Universitario de Fuenlabrada, Spain

$\mathrm{b}$ Department of Nuclear Medicine, CIBIR, Logroño, Spain

c Department of Medical Physics, Hospital Universitario Doce de Octubre de Madrid, Spain

d Department of Nuclear Medicine, Clínica Universidad de Navarra, Pamplona, Spain

e Department of Nuclear Medicine, Hospital General Universitario de Ciudad Real, Spain

${ }^{\mathrm{f}}$ Department of Medical Physics, Hospital Universitario La Paz de Madrid, Spain

g Department of Radiation Oncology, Hospital Ramón y Cajal de Madrid, Spain

$\mathrm{h}$ Department of Radiation Oncology, Hospital General Universitario de Ciudad Real, Spain

${ }^{i}$ Department of Medical Physics, Hospital Universitario de Fuenlabrada, Spain

\section{A R T I C L E I N F O}

\section{Article history:}

Received 11 September 2012

Accepted 11 October 2012

\section{Keywords:}

Radiotherapy planning

PET

PET-CT

CT

Delineation

Contouring

\begin{abstract}
A B S T R A C T
Positron emission tomography (PET) with ${ }^{18} \mathrm{~F}$-fluorodeoxyglucose (FDG) is a valuable tool for diagnosing and staging malignant lesions. The fusion of PET and computed tomography (CT) yields images that contain both metabolic and morphological information, which, taken together, have improved the diagnostic precision of PET in oncology. The main imaging modality for planning radiotherapy treatment is CT. However, PET-CT is an emerging modality for use in planning treatments because it allows for more accurate treatment volume definition. The use of PET-CT for treatment planning is highly complex, and protocols and standards for its use are still being developed. It seems probable that PET-CT will eventually replace current CT-based planning methods, but this will require a full understanding of the relevant technical aspects of PET-CT planning. The aim of the present document is to review these technical aspects and to provide recommendations for clinical use of this imaging modality in the radiotherapy planning process.
\end{abstract}

@ 2012 Published by Elsevier Urban \& Partner Sp. z o.o. on behalf of Greater Poland Cancer Centre.

\footnotetext{
* Corresponding author.

E-mail address: begocaba@gmail.com (B. Caballero Perea).

1 On behalf of the Spanish Society of Radiation Oncology (SEOR), Spanish Society of Nuclear Medicine and Molecular Imaging (SEMNiM), and the Spanish Society of Medical Physics (SEFM). 


\section{Introduction}

\subsection{The radiotherapy process}

Radiotherapy (RT) has long played an important role in treating and curing cancer. The effectiveness of RT is dose-dependent: the higher the dose and the larger the volume irradiated, the greater the probability of controlling the cancer. However, as the dose increases, so does the likelihood of damaging healthy tissues. For this reason, the aim of treatment is to maximize the dose delivered to the tumour while preserving, to the extent possible, adjacent healthy tissue. In short, like other treatments, the aim is to achieve the highest cure rate with the fewest side effects.

Radiotherapy treatment is a complex process involving a series of sequential steps that make up what is known as the radiotherapy process. In Spain, quality control criteria for RT have been established by law (Royal Decree 1566/1998, July 17). These criteria define and regulate the stages in the radiotherapy process, as follows: initial diagnosis and patient evaluation, therapeutic decision, tumour localization, irradiation treatment plan, treatment simulation, treatment delivery and quality control, and final assessment and follow up. Fig. 1 shows the process schematically. ${ }^{1}$

Once the therapeutic decision has been made and the patient has been deemed suitable for radiotherapy, the next step is to locate the target volume to be irradiated. In most developed countries (including ours), localization is performed via three-dimensional (3D) computed tomography (CT). At present, irradiation treatment planning is based on the electron densities measured by CT imaging; for this reason, there is currently no substitute for CT-based planning. Once the CT images have been acquired-under specific conditions that can be reproduced in future treatment sessions-the radiation oncologist uses the data to delineate the gross tumour volume (GTV), also called the macroscopic tumour volume. ${ }^{2}$ The GTV is the demonstrable extension and localization of the malignant growth. Three types of GTV are possible: primary GTV (primary tumour), nodular GTV (metastatic lymph nodes), and metastatic GTV (distant metastasis). Contouring of the GTV is based on data obtained from imaging (CT, magnetic resonance image [MRI], etc.), clinical examination, and tumour stage. According to the International Commission on Radiation Units and Measurements (ICRU) Report No. $83,{ }^{1}$ tumour staging should be based on the TNM/AJCC or UICC staging systems and the ICD-O (International Code for Disease in Oncology by the World Health Organization). ICRU 83 also recommends that the imaging technique(s) used for diagnosis (e.g., CT, MRI T2, FDG-PET, F-MISO-PET, etc.) be recorded, in addition to the exact point in time during treatment (e.g., at diagnosis, at $45 \mathrm{~Gy}$, etc.) at which delineation was performed.

Once the GTV has been contoured, a margin must be added to the GTV volume to account for the likely presence of subclinical disease, which must also be irradiated. This volume (GTV + margin) is called the clinical target volume (CTV). An additional margin, called the internal margin (IM), must then be added to the CTV. The IM is necessary to account for variations in the position, size, and shape of the tumour that inevitably occur during and between treatment sessions. An additional margin, known as the set-up margin (SM), must be added to compensate for imprecision and errors in reproducing patient positioning and in aligning the therapeutic beams during acquisition of images for planning purposes and over the course of treatment. The combination of all these margins $(\mathrm{CTV}+\mathrm{IM}+\mathrm{SM})$ results in the planning target volume (PTV), a geometric concept used for treatment planning. The PTV will then be used to select the size and appropriate configuration of the beams to assure that the prescribed dose is actually delivered to the CTV. Finally, we need to delineate the organs at risk (OAR), which also requires an additional margin to define the planning risk volume (PRV) of the OARs. The purpose of the PRV is to predict possible complications in healthy tissue. Once the PTV has been designed and the dose prescribed, the radiation oncologist will then establish dose restrictions to the PRV. Once this has been done, the medical physicist will design an irradiation plan that fulfils all these conditions and will send the plan to the radiation oncologist for approval. It is the responsibility of the radiation oncologist to assure that the treatment is applied and verified correctly.

Modern planning and treatment delivery systems are capable of producing irradiation fields that deliver the prescribed dose to the tumour volume with an extraordinary degree of precision. ${ }^{3}$ Such techniques-known as conformal RT-utilize medical images (generally, CT-based images) to develop the RT treatment plan. Conformal RT also relies on advanced computing tools to perform the complex dosimetric calculations and to implement strict geometric and dosimetric control procedures in order to minimize errors.

\subsection{The value of PET-CT in radiotherapy}

Positron emission tomography (PET) with ${ }^{18} \mathrm{~F}$-fluorodeoxyglucose (FDG) plays a very important role in the diagnosis and staging of malignant lesions, particularly when hybrid PET-CT imaging studies are used. ${ }^{4}$ The fusion of metabolic and structural imaging studies has dramatically improved the diagnostic precision of PET in oncology. ${ }^{5}$

Although FDG-PET was not originally designed for planning radiation therapy, evidence to date indicates that planning is one of the most promising indications for this imaging modality. ${ }^{6}$ According to the International Atomic Energy Association (IAEA), RT planning requires the use of precise diagnostic methods to assess disease spread, and PET-CT is one of the most accurate methods currently available. For this reason, PET-CT is of great use in planning RT, particularly for tumour locations in which it has already proven its value. ${ }^{7}$ The main benefit of PET-CT in RT treatment planning is its ability to better define the treatment volume. The use of PET-CT in RT planning has many potential benefits: it facilitates contouring, reduces inter-observer variability in geometric planning, and, most importantly, can improve the therapeutic efficacy. Similarly, it may also have an impact on the treatment indication (i.e., curative or palliative). Although the value of PET-CT for RT planning is still being investigated, it seems highly probable that this new technology will have a large, positive impact on treatment planning in the very near future and it may eventually replace current CT-based methods. ${ }^{8}$ 


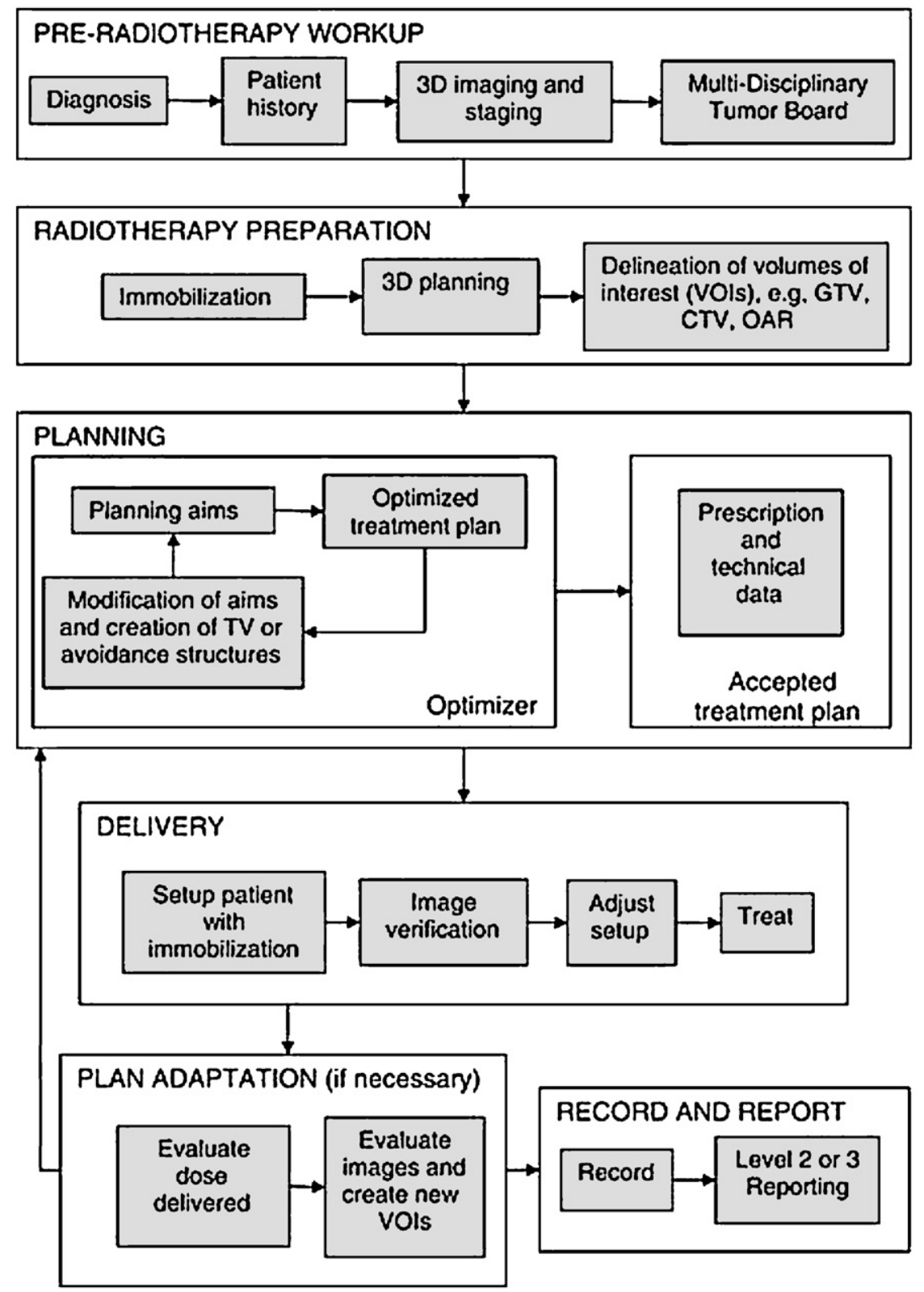

Fig. 1 - Schematic diagram of the radiotherapy process.

Reprinted from ICRU Report 83: Prescribing, Recording, and Reporting Photon-Beam Intensity-Modulated Radiation Therapy (IMRT). Volume 10, Issue 1, April 2010 by permission of Oxford University Press.

\subsection{The need for a multidisciplinary group and some} general recommendations

FDG-PET-CT imaging for RT planning is one of the most complex diagnostic imaging examinations currently available. Before this imaging modality can be used in therapeutic applications, we must first fully understand its limitations and develop standardize protocols for implementation. Importantly, if we fail to standardized procedures, it is likely that results will be unsatisfactory. Although FDG-PET-CT may one day become standard clinical practice, we must first evaluate the relevant technical aspects of this technique in RT planning. The purpose of the present document is to review the technical facets of the procedure and to provide recommendations for clinical use.

In the image acquisition process of PET-CT for RT planning, radiation oncologists and nuclear medicine physicians need to work together in close collaboration. This partnership is crucial due to the enormous organizational effort required and to assure correct interpretation of the imaging studies. Medical physicists also play an essential role due to their involvement in standardizing the process and in quality control. For all these reasons, multidisciplinary collaboration between radiation oncologists, medical physicists, and nuclear medicine physicians is absolutely essential if PET-CT imaging is to fulfil its potential for RT planning. 
Recommendations on the use of PET-CT in RT planning have been published previously, including those described by the European Society of Radiation Oncology (ESTRO) and European Association of Nuclear Medicine (EANM) in a special issue published in the journal Radiotherapy \& Oncology in 2010. ${ }^{9}$ The ESTRO-EANM special issue contains much valuable information and we highly recommend it to our readers.

The present document provides technical recommendations for carrying out RT planning based on ${ }^{18} \mathrm{~F}$-FDG PET-CT in adults. This document is the result of a consensus agreement developed by a working group comprised by members of the Spanish Society of Radiation Oncology (SEOR), the Spanish Society of Nuclear Medicine and Molecular Imaging (SEMNiM), and the Spanish Society of Medical Physics (SEFM). The ultimate aim of this working group is to combine our efforts to obtain the maximum benefit from combined metabolic and morphological imaging in the field of radiation oncology. The recommendations described here can be used by cancer treatment centres that are considering implementing PET-CT imaging in RT treatment planning. An important aim of this consensus document is to standardize the process in order to facilitate comparison of results between different centres.

\section{Required equipment}

The CT simulator is the tool commonly used to acquire images for RT planning, in a process known as CT simulation (or virtual simulation). It is called simulation because images are acquired with the patient in a simulated treatment position that mimics the position that will eventually be used to delivery radiation therapy. A CT simulator includes:

- a CT with a specially adapted table (a firm flat tabletop) that mimics treatment conditions,

- an alignment-marking system that uses external lasers,

- a high-capacity workstation for image manipulation,

- a computer software package to run the virtual simulation.

In order to use FDG-PET-CT in RT planning, the PET-CT scanner must first be converted into a PET-CT simulator. This transformation requires the following elements:

- Flat table top made of carbon fibre with indentations in the edges for indexing the immobilization devices. This couch top should be identical to the one used in the treatment unit.

- A system of external lasers to align the patient and to indicate where the reference marks should be placed on the patient's body. The laser system, generally a group of three beams-two lateral beams for the coronal plane and a beam located on the ceiling to indicate the sagittal plane-can either be fixed or mobile. Likewise, the movements of the lasers can either be manually or automatically programmed via connection to the virtual simulator console.

- The virtual simulation console, generally located in the Radiation Oncology Department, requires special tools to visualize the metabolic images that are co-registered with CT-based images, as well as tools for semi-automatic segmentation of the metabolic images.

\subsection{Gantry aperture of the PET-CT}

At present, most PET-CT simulators in current use have one key drawback that impedes their ability to precisely reproduce conditions in the treatment unit (i.e., linear accelerator): the distance between the scanner and the body surface. The gantry aperture of most PET-CT simulators is only $70 \mathrm{~cm}$, notably less than the $\geq 85 \mathrm{~cm}$ in CT simulators. Recently, however, new hybrid devices have been introduced to the market with extra-large apertures of $85 \mathrm{~cm}$. As more centres purchase these devices, this issue will no longer be pertinent.

A larger gantry aperture facilitates patient positioning under treatment conditions. In addition, a wide scan field of view (SFOV) assures that the entire delineated volume is contained within the limits of the image, an essential condition for the use of axial images in RT planning. Conventional scanners (gantry aperture $=70 \mathrm{~cm}$ ) provide a maximum SFOV of $48-50 \mathrm{~cm}$ and this can be problematic, particularly in contouring tumours in large patients, as the small CT FOV causes some CT projection views to be truncated, and thus not completely visualized. The extended FOV, or extrapolated data function, permits image reconstruction by widening the FOV. However, it is important to keep in mind that objects located in that part of the extrapolated image may be geometrically distorted and thus the Hounsfield units may be imprecise-and either of these factors could lead to errors in dosimetric calculations. ${ }^{10}$ For this reason, great caution must be taken when using extrapolated images in RT planning. An alternative would be to obtain a new series of CT images with a wider FOV and then co-register these with PET-CT images in the treatment planning system (TPS).

\section{Interdepartamental organization}

Given that the PET-CT procedures for RT planning require the participation of multiple departments, coordination and collaboration are essential. This multidisciplinary focus requires that all specialists fully understand the critical aspects of the whole process-even those aspects that are theoretically the responsibility of other disciplines-in order to correctly apply PET-CT for RT planning.

The first step is to select the tumour locations that are considered suitable for PET-CT planning. It is important to define all the steps in the process, starting from the moment the patient is first evaluated by the Department of Radiation Oncology. Given that metabolic avidity varies by tumour and localization, it is recommended that all factors related to a particular tumour localization be evaluated before other locations are considered.

Once consensus has been reached in terms of the methodology, the next step is to verify the availability of all necessary equipment and material (laser, flat table top and immobilization devices compatible with those used in the Radiation Oncology Department). It is also important to verify compatibility and connectivity between the workstations used in the nuclear medicine and radiation oncology departments.

PET-CT planning has an added benefit apart from improved contouring: it offers an ideal opportunity to reassess the original tumour staging. Most patients undergo diagnostic CT (with 
intravenous contrast) for staging purposes. However, PET-CT can detect previously unsuspected distant disease in approximately $20 \%$ of the most common cancers; as a result, the addition of PET-CT often leads to changes in staging. In fact, this aspect of PET-CT further supports its routine application in order to optimize patient selection. ${ }^{11}$ All patients should undergo a standard PET-CT imaging from the orbitomeatal line to the upper third of the lower extremities before being included in the RT planning protocol. It is not necessary to repeat the planning PET-CT if patients have undergone a prior diagnostic PET-CT in the previous 4 weeks.

To reduce costs, delays, and irradiation, standard PET-CT (to scan for distant metastasis) should ideally be performed on the same day as the planning PET-CT. Once distant metastasis has been ruled out, the planning PET-CT can be scheduled (according to an established protocol) to coordinate the work of the technologists, operators, and nurses involved in patient positioning, PET-CT acquisition, and intravenous contrast administration.

The PET imaging study should be performed from 3 to $5 \mathrm{~h}$ following administration of ${ }^{18}$ F-FDG to take advantage of differences in uptake between tumour and normal tissues. The contrast between normal and tumour tissues is at its peak during this time period. Said another way, the relative metabolic activity of ${ }^{18} \mathrm{~F}-\mathrm{FDG}$ in tumour tissues is at its greatest from 3 to $5 \mathrm{~h}$ following administration. ${ }^{12}$

Planning PET-CTs require at least $30 \mathrm{~min}$ more than diagnostic PET-CTs due to patient positioning, use of immobilization devices and masks, and in some cases catheterization, tattoos, preparation of the contrast injector (in cases in which the CT images are of diagnostic quality) and acquisition of at least 2 fields.

The imaging study will need to be evaluated by a physician from nuclear medicine or radiation oncology to ensure image quality. After the pathological lesions have been defined, the radiation oncologist can then select the areas to be treated. It is important to record the criteria (i.e., metabolic and/or morphological data from the PET and/or CT) used to select the therapeutic volume, as it is vital to know what data was used to include (or exclude) a lesion in the treatment field. These criteria should be recorded in accordance with the norms required by ICRU 83 (described in the Introduction section). This is important because it allows us to assess, during follow up, the role of each technique (PET and CT) in selecting the correct field to be treated. Moreover, this allows us to evaluate the association between the imaging technique and possible loco-regional recurrence and patient survival.

\subsection{Strategies for the use of PET-CT in RT planning}

Various strategies exist for the use of PET-CT in planning RT. These are summarized, from least to most complex, below:

(a) PET-CT without intravenous contrast. The patient is positioned on the flat table top, similar to treatment positioning, but without immobilization devices. The PET-CT images are used to complement those obtained in the CT simulation. Both sets of images need to be co-registered. This method only requires a small amount of extra time in the PET-CT room. (b) PET-CT with intravenous contrast. The patient is positioned on the flat tabletop in the same position that will be used for treatment, and immobilized by the same devices that will be used during treatment (these devices should be prepared previously in another room). Likewise, the reference marks for daily repositioning should also be applied. The PET-CT images can then be transferred to the TPS for planning. This strategy requires the presence of an RT technologist in the PET-CT room and some extra time, but no more than an additional $30 \mathrm{~min}$ at most.

(c) The entire process-simulation plus preparation of immobilization devices-is performed in the PET-CT room, after which the images are transferred to the TPS. This strategy requires the presence of a RT technologist in the PET-CT room and some extra time, at least 30 min compared to a diagnostic PET-CT.

Strategy "a" is the simplest and least burdensome in terms of the extra time needed. The downside, however, is that the geometric uncertainties of this approach limit the utility of the metabolic images; in other words, the images can be used to localize the macroscopic lesions but not for contouring. Strategy "c", in contrast, is the most time-consuming and could disrupt the work of a PET-CT team whose primary objective is clinical diagnosis. Very few radiation oncology departments (none in Spain) have a dedicated PET-CT for RT planning and, realistically, strategy "c" requires a dedicated PET-CT. An additional drawback is greater exposure of staff to the radiation emitted by the isotope.

Strategy "b" is moderately demanding in terms of its impact on the PET-CT workload but it is acceptable for the Nuclear Medicine Department as long as the number of patients scanned is not excessive. In addition, the images obtained in this scan can be directly used for planning radiotherapy treatment. Given that PET-CT scanners are usually located in the Department of Nuclear Medicine, we believe that this strategy is the least burdensome on the department without compromising the geometric precision required for planning.

\subsection{Patient preparation: the role of radiation oncology}

\subsubsection{Patient positioning}

The precision of radiation therapy depends in large part on proper patient positioning and on the ability of the team to accurately re-position the patient on a daily basis throughout the course of treatment. Positioning will also depend on the tumour location. Table 1 shows the recommended positions by tumour localization.

Several factors can impede correct patient positioning, including (a) physical deterioration caused by the cancer; (b) presence of comorbidities, and (c) patient pain or discomfort. For example, dysphagia due to tumour growth in the oropharynx or hypopharynx will cause an accumulation of saliva in the mouth and oropharynx, thus making it difficult for the patient to remain supine with the neck extended during the time needed to acquire the PET images. Similarly, supine positioning is not possible in patients with respiratory or cardiac comorbidity due to intolerable dyspnoea. Pain can also limit the patient's ability to remain in the proper position. Nevertheless, undesired movement due to pain can be limited or 
Table 1 - Position of patient according to tumour location.

\begin{tabular}{llll} 
Tumour location & \multicolumn{1}{c}{ Trunk } & Neck & \multicolumn{1}{c}{ Extremities } \\
\hline Head and neck & Supine & Extension & Arms along the length of the body; caudal retraction of the shoulders \\
Lung & Supine & Neutral & Arms flexed above the head \\
Upper abdomen & Supine & Neutral & Arms flexed above the head \\
Pelvis-rectum & Prone/supine & Flexion & Arms in front of the head \\
Pelvis-cervix & Supine & Neutral & Arms crossed over the chest \\
\hline
\end{tabular}

prevented by prescribing more analgesics to control pain during simulation.

\subsubsection{Patient alignment}

Whatever the patient position, his or her longitudinal axis should be parallel to the longitudinal axis of the PET-CT table, with no lateral rotation around the axial plane. To avoid axial rotation and to align the patient properly, the room should be equipped with one sagittal and two lateral laser beams (one on each side of the room), and all three beams should be aligned with the scanner axis. ${ }^{10}$ The sagittal laser projects a narrow beam (approximately $1 \mathrm{~mm}$ in diameter) in the form of a fan projected onto the skin of the patient in a line that should coincide with centre of the patient's body through the anterior side of the head, thorax and abdomen. The lateral lasers project two narrow orthogonal beams onto the patient's skin to form two perpendicular lines that define the axial and coronal planes. The points where these laser beams intersect are called triangulation points. The radiotherapy technologist should place the triangulation points in the anatomical area of interest for treatment, and should either tattoo the patient's skin at the precise location of these points or mark the immobilization devices (e.g., thermoplastic masks) if the triangulation points happen to be coincide with the position of the immobilization device. For alignment reproducibility, the skin (or immobilization device) should be tattooed (marked) at an additional point on the sagittal plane.

\subsubsection{Patient immobilization}

In a perfect world, the object scanned for RT planning would be solid, rigid (i.e., non-deformable), and immobile. Unfortunately, the human body is none of these: it is deformable (both externally and internally), and mobile (both voluntarily and involuntarily). For this reason, a variety of immobilization devices are used to reduce both voluntary and involuntary movements during image acquisition and treatment delivery. It is highly recommended that these devices be attached to the flat table top and also that they be indexed (referenced to a fixed position on the table) to assure precise reproducibility of positioning during planning and treatment. The use of immobilization devices during image acquisition has proven useful in reducing movement artefacts, but these devices must be indexed and attached to the table to prevent lateral, longitudinal, or rotational movements.

3.2.3.1. Indexed flat table top. The table tops placed over the RT treatment tables are flat (to facilitate repositioning), narrow (to allow the gantry to turn around the couch), and constructed of rigid, low-density materials (to prevent buckling under the weight of the patient). Generally they are made of carbon fibre, an extremely strong yet low-density material.
3.2.3.2. Immobilization devices. The main aim of immobilization devices is to avoid involuntary patient movement during treatment. However, they are also useful in PET-CT image acquisition since patient movement between the transmission (CT) and emission (PET) scans will cause errors in attenuation correction, thereby creating artefacts. ${ }^{13}$ As mentioned previously, the devices should be attached and indexed to the PET-CT table to avoid movements and should also be small enough to prevent collisions with the gantry and to avoid producing artefacts.

3.2.3.3. Monitoring involuntary movements. It is important to keep in mind that patients with head \& neck or lung tumours tend to cough frequently and may have deglutition difficulties, both of which increase the likelihood of involuntarily movements during the PET-CT scan. In these cases, the patients should be carefully monitored to prevent artefacts of movement, or even interruption of the imaging study. For similar reasons, all individualized immobilization devices should be prepared beforehand (ideally in an adjacent room) to avoid prolonging the time spent in the PET-CT.

\subsection{Patient preparation: the role of the nuclear medicine department}

The preparation and administration of the FDG dose, and image acquisition should follow the procedures set forth by the EANM and SEMNiM, given here as follows. ${ }^{14}$

\subsubsection{Before the appointment}

- Recommendations by the Department of Radiation Oncology should be followed regarding pre-study preparation (e.g., laxatives for patients with rectal cancer, thermoplastic masks for patients with head \& neck cancer, etc.).

- A brief questionnaire should be given to the patient before the PET-CT appointment in order rule out possible contraindications.

- The possibility of pregnancy should be evaluated in patients of child-bearing age. In case of doubt, the patient should be asked to have a pregnancy test and to bring the results on the day of the PET-CT appointment. For women who are breast-feeding, feeding should be interrupted for $24 \mathrm{~h}$ following FDG administration.

- Patients should fast for 4-6h prior to the appointment. This restriction includes ingestion of candy, gum, and sugary drinks. It is highly recommended that patients drink water during this period.

- No physical exercise is permitted in the $24 \mathrm{~h}$ period before the test. 
- Patients should avoid driving a car and other stressful activities on the day of the appointment. It is recommended that the patient be accompanied by a family member.

- Patients should drink 2-3 glasses of water and take their usual medications on the day of the appointment.

- Diabetic patients: patients with diabetes should be asked to provide details about their treatment, type of diabetes, and typical blood glucose levels. The patient should be told to fast prior to the appointment, which should be scheduled early in the morning.

- In cases in which a CT with intravenous contrast is required, the usual recommendations should be followed after verifying that the patient has no contraindications for the test (see below*).

\section{(*) Contraindications}

- Absolute contraindications

- Known or suspected allergy to iodine contrasts.

- Hypothyroidism. Patients with thyroid disease and risk of developing thyrotoxicosis should be evaluated by an endocrinologist before and after administration of iodine contrast:

- Relative contraindications

- Renal insufficiency. Intravenous contrast can be given as long as the patient is adequately hydrated and the contrast dose is reduced. Administration of nephrotoxic drugs, dopamine, mannitol and diuretics should be avoided. There is no need to coordinate with dialysis.

- Treatment with the oral antidiabetic drug, metformin (a biguanide). Either of the following two procedures can be used:

Procedure A:

- Administration of metformin should be suspended $24 \mathrm{~h}$ before intravenous contrast administration and restarted no sooner than $48 \mathrm{~h}$ after imaging and only after serum creatinine levels have been checked to assure normal kidney function.

- In patients with suspected renal function impairment (DM > 60 years with HTA) or altered serum creatinine levels, metformin should be suspended $48 \mathrm{~h}$ before the study, and not restarted until $48 \mathrm{~h}$ after (with prior assessment of serum creatinine to assure absence of renal function impairment).

- In both of these approaches to Biguanides management, the Biguanides should be temporarily replaced with an alternative medication prescribed by an endocrinologist.

Procedure B:

Intravenous contrast should not be administered to outpatients being treated with Biguanides who have creatinine values greater than $1.3 \mathrm{mg} / \mathrm{dl}$.

\subsubsection{During outpatient stay in the nuclear medicine} department

- The nurse in charge should be notified immediately upon the patient's arrival at the department. The nurse should then notify the technologist, who will set the time for the image acquisition and contact the supervising physician.
- Patient data should be verified before any intervention is performed.

- The physician or nurse in charge should administer a brief questionnaire to specifically review current medications, fasting status, allergy history, and any contraindications to muscle relaxants or intravenous contrast agents.

- Should insulin be required, the following guidelines can be used:

- Blood glucose, $200-250 \mathrm{mg} / \mathrm{dl}$ : administer 4 units of rapidacting insulin.

- Blood glucose, 250-300 mg/dl: administer 6 units of rapidacting insulin.

- Blood glucose $>300 \mathrm{mg} / \mathrm{dl}$ : notify the physician in charge.

If an insulin shot is given, FDG administration should be delayed by at least $2 \mathrm{~h}$ because hyperinsulinaemia could lead to increased glucose uptake in the muscles relative to other tissues.

- The patient should remain supine on the couch, covered by a blanket, in semi-darkness without any lighting or auditory stimulation for 45-60 min. The patient should not speak nor chew gum. However, if the patient needs to urinate, this should be permitted.

- Examinations of the pelvic area may require insertion of a catheter to fill the bladder with $200-250 \mathrm{ml}$ to assure reproducibility of the adjacent pelvic structures. The same amount of liquid should be used throughout the planning process. Catheterization should be performed prior to final positioning and before acquisition of the PET-CT image.

\subsubsection{Administration technique}

- Intravenous injection with syringe protector. Avoid administering the contrast through catheters and permanent devices.

- With the patient in a seated position, start an intravenous cannulation using a three-way stopcock. Prepare a connection to physiological saline solution.

- Verify cannula permeability by injecting 5-10 $\mathrm{ml}$ of physiological saline solution before and after FDG administration, making sure to prevent extravasation at all times.

- The physician in charge should be immediately notified of any incidents that occur during injection; if relevant, these incidents should be noted on the data collection sheet. If extravasation occurs, this should be recorded on the data collection sheet and the procedure can continue without interruption.

- A total of 500 cc of physiological saline should be administered during the first 50 min of the waiting period.

- In patients with breast or lymph node lesions, the radiopharmaceutical should be administered in the contralateral extremity.

- The cannula should be removed and the patient instructed to urinate $45 \mathrm{~min}$ after administration has been completed. If intravenous contrast is used, only the 3-way stopcock need be removed. 
3.3.4. Protocol for administering radiological contrast agents (based on recommendations of the Spanish Society of Medical Radiology [SERAM] $)^{15}$

Oral contrast media is preferred when acquiring CT images for RT planning because it improves visualization of the digestive tract. Intravenous contrast media, on the other hand, can be used to enhance tumour visibility and to identify vascular structures. However, contrast media can generate artefacts on the PET images if CT-based attenuation correction (CTAC) is performed after administration. ${ }^{16}$ The problem is relevant if the radioactivity concentration of the contrast agent surpasses radiodensity levels of $200 \mathrm{HU} .{ }^{17}$ Even though the increase in standardized uptake values (SUV) in areas with high contrast concentrations is significant when CTAC is performed, this increase is not clinically relevant. The use of low-density neutral oral contrast material (barium sulfate $0.1 \%$ ) does not produce clinically detectable errors in PET studies corrected for attenuation. Moreover, contrast agents have been used in planning PET-CT in various anatomical locations without any apparent effect on clinical results. ${ }^{18}$

\section{Oral contrast}

- In all cases, water should be used as a negative oral contrast.

- Administration protocol

- Administer 600cc (2-3 glasses) of water orally before injecting the radioisotope.

- Give 250cc (1 glass) of water to the patient to drink upon entering the PET-CT examination room

Intravenous contrast (SERAM recommendations). Particular attention should be paid to the permeability of the IV cannula used to infuse the contrast agent. The infusion pump should also be checked to assure that it is working properly. Any extravasation or inadequate infusion could invalidate the procedure by compromising the quality of the CT images or by causing patient movement due to pain at the insertion point. A test bolus with saline solution should be administered (all modern contrast media injectors have this capability).

- Use an iodinated contrast agent (nonionic iomeprol or similar) delivered through an injector.

- Use the same peripheral cannula for administration of FDG.

- Check the system and prepare the contrast infusion according to the protocol established by nursing.

- The contrast dose varies depending on the imaging study to be performed and the patient's weight. For adults, the range is usually between $80 \mathrm{ml}$ and $120 \mathrm{ml}$.

- Timing of administration:

- The contrast agent should be administered during acquisition of the CT data for attenuation correction.

- The delay in image acquisition will depend on the specific tumour localization: normally, 20-30s for head \& neck tumours and $60 \mathrm{~s}$ for others, although this protocol can vary depending on preferences.

\section{Image acquisition}

\subsection{Time, field of study, pre-positioning, and} preparation for image acquisition ${ }^{19,20}$

The interval between FDG injection and image acquisition (i.e., the uptake period) is perhaps the most critical parameter of the process. In diagnostic PET-CT, a whole body scan is usually performed at $60 \mathrm{~min}$ post-FDG administration. In contrast, for RT planning, the scan should be performed at least $3 \mathrm{~h}$ after FDG injection because tumour detection sensitivity increases over time. FDG concentration in cancerous tissue will vary by tissue type, but concentration levels increase in all types for at least $90 \mathrm{~min}$ after injection. Unfortunately, it is not possible to compare the results of most case series that have been published to date due wide variations among these studies in the time at which SUV data were measured. To avoid this problem in the future, we recommend that variation in uptake time from patient to patient be limited to less than $10 \mathrm{~min}$. In clinical practice, this requires careful planning between FDG administration and the start of imaging. SUV is a stable and highly reproducible parameter, but only when strict protocols are followed for data acquisition and analysis.

Patients should be instructed to empty their bladder before the scan to limit radiation to the urinary system. All metallic objects must be removed whenever possible. A flat table top similar to the one used for treatment is recommended. The patient should be carefully placed in the same position for both simulation and treatment; this alignment process should be guided by laser beams and the skin markings that define the treatment area. ${ }^{21}$ The same immobilization devices should be used for both simulation and treatment. If thermoplastic moulds have been created, these same moulds should be used for positioning.

To check for distant metastasis, we recommend scanning from the skull base to the proximal third of the thigh. For tumours with a high affinity for the scalp, skull or brain, the head should be included in the field. For optimal evaluation of the head and neck region, the arms should be extended along the side of the body. Similarly, for the thorax-abdomenpelvis, patients should be positioned with their arms elevated above the head to avoid beam-hardening artefacts and artefacts caused by truncation of the FOV on the CT image. Pelvic imaging should be performed with the patient in prone position. Patients with rectal cancer should have a full bladder.

Generally, image acquisition is performed by moving the table top through the detection field until the relevant areas have been scanned. Depending on the model of the tomographic scanner, the axial detection field measures from 15 to $25 \mathrm{~cm}$, thus requiring from 5 to 10 table top movements to examine the entire body. The mean duration for each table top position can range from 2 to $5 \mathrm{~min}$.

\subsection{CT protocol ${ }^{22}$}

CT is a valuable tool with many possible uses: attenuation correction, localization of hypermetabolic lesions, volume contouring in RT planning, and, of course, for diagnostic purposes. When CT is used for attenuation correction, the 
milliampere-second setting is low (40-50 mAs) to reduce the radiation dose to the patient. In contrast, a much higher setting (>120 mAs) is needed to acquire images of sufficient quality to enable contouring without the need for contrast media.

Diagnostic CT imaging is performed with intravenous contrast and tube current modulation. The use of intraluminal contrast provides adequate visualization of the gastrointestinal tract and the contrast agent can be positive (diluted barium) or negative (water). Because high barium concentrations can produce an attenuation artefact with a significant overestimation of ${ }^{18} \mathrm{~F}$-FDG concentration, the barium must be diluted to assure image quality.

- Acquisition of the topogram. The first step in image acquisition in a PET-CT scanner is to acquire a topogram (overview scan). This image is obtained with the x-ray tube locked in a fixed position-typically a frontal position, although other positions are possible. The topogram is obtained with the table top in continuous movement in order to acquire an anatomical image that reveals the distinct structures; it is this image that the operator will use to define the axial extension of the PET-CT image. Once the limits of the imaging study have been determined, the acquisition fields of both (PET and CT) should be adjusted such that the acquisition of the CT (with continuous movement of the table top) coincides in extension with that of the PET scan (with discrete movements of the table top). In some PET-CT scanners, the FOV of the CT and PET differ (e.g., $50 \mathrm{~cm}$ for CT and $60 \mathrm{~cm}$ for PET), leading to differences in the images. In this case, it is important to verify that the image with the smaller FOV (normally the CT) includes all relevant body regions. If left uncorrected (i.e., by failing to reposition the patient), these differences in FOV can cause truncation artefacts on the reconstructed images.

- CT acquisition. This is performed in the standard way, with a specific breathing protocol during image acquisition (smooth and superficial breathing) designed to help make the CT and PET images coincide more precisely. Various parameters can affect the time needed to acquire the CT image: the size of the anatomical region of interest; the number of slices of the CT scanner; the tube rotation speed; and the speed at which the table top is moved. Whole body imaging usually takes less than 1 min. Artefacts produced by respiratory movements are especially relevant in lesions located at the base of the lungs and in the liver dome. For this reason, depending on the resources available, an additional inspiratory chest CT can be performed to evaluate lung nodules, or for 4D PET-CT.

\subsection{Protocol for PET emission scan}

Once the CT study has been completed, the table top can be moved in order to position the patient in the FOV for the PET tomography. The PET-CT operator will decide when to begin the examination as well as the direction of the table top. When it is important to limit the artefact produced by radiopharmaceutical accumulation in the bladder, a caudocranial orientation is used. PET imaging is performed by acquiring the sinograms in the various table top positions that comprise the field examined in the CT. The total duration of the PET scan depends on the number of table top positions and the time allotted for the scan at each position. Depending on the tomographic model (2D or 3D), the total duration ranges from 6 to $35 \mathrm{~min}$. Late PET imaging-performed between 90 and $180 \mathrm{~min}$ following FDG injection-improves the lesion-to-background ratio, thus facilitating image interpretation.

\subsection{Dosimetry}

In FDG PET-CT studies, the effective dose of radiation delivered to the patient is the sum of the radiopharmaceutical dose (approximately $10 \mathrm{mCi}$ of ${ }^{18} \mathrm{~F}$-FDG [6-7 mSv]) and the CT dose (2-4 mSv), with a typical total dose of approximately $10 \mathrm{mSv}^{23}$ The CT dose is highly variable depending on the protocol and systems used, and dosimetric optimization can reduce the dose. The effective dose ranges from 2 to $80 \mathrm{mSv}$ but this must be calculated for each system and protocol.

For ${ }^{18} \mathrm{~F}$-FDG, the effective doses per unit of activity administered $(\mathrm{mSv} / \mathrm{MBq})$ are as follows ${ }^{24}$ :

\begin{tabular}{llllll}
\hline Radiopharmaceutical & Adult & $\begin{array}{l}15 \\
\text { years }\end{array}$ & $\begin{array}{l}10 \\
\text { years }\end{array}$ & $\begin{array}{l}5 \\
\text { years }\end{array}$ & 1 year \\
\hline${ }^{18}$ F-FDG & 0.0183 & 0.0233 & 0.0356 & 0.0534 & 0.0927
\end{tabular}

\section{Interpretation criteria for metabolic images}

Interpretative criteria for FDG-PET for diagnosis of tumours have been widely described in the literature. ${ }^{14}$ In this section, our aim is primarily to provide the reader with a reflexive (rather than objective) approach to interpretative criteria. We include these tentative criteria to help develop consensus recommendations for PET planning in radiotherapy. By no means are these criteria to be considered definitive.

The sensitivity and specificity of PET vary by tumour type and each case must be considered on its own. This, of course, has implications for RT planning. In general, if the aim is to treat the whole tumour, it is best to use interpretative criteria that have a high sensitivity (assuming a low specificity), with the understanding that non-cancerous tissue (i.e., healthy tissue) will likely be included in the radiotherapy field. Such an approach is appropriate for treatments with curative intent. For example, if anomalous tracer deposits are found in regional or distant lymph nodes, these should be considered positive and included in the treatment volume. In contrast, if our main objective is to reduce morbidity by minimizing radiation to healthy tissues, then the interpretative criteria should have a greater specificity-even though this implies an increased risk of relapse. In such cases, all relevant diagnostic procedures (including biopsy) should be performed to rule out or confirm the positive PET findings before the therapeutic approach is planned.

The sensitivity and specificity of FDG-PET is slightly better than conventional morphological imaging exams for nodal staging in head \& neck tumours, although the diagnostic differences between functional and morphological imaging are not statistically significant ${ }^{25}$ in this case. Even so, PET imaging 
is valuable as a complementary technique with a potential role in RT planning. The potential advantages of FDG-PET are as follows: a decrease in interobserver variability in contouring the GTV; a smaller GTV in most cases; identification of active disease that remain undetected by other techniques (CT and/or MRI); and the ability to identify areas of the GTV that might require an additional dose of radiotherapy. ${ }^{26}$ Other, more specific benefits of FDG-PET include GTV contouring via automated segmentation methods, and definition of the isodose distribution to the primary tumour. Preliminary data also suggest that PET may have a promising role in adaptive treatments and in patient follow-up.

The sensitivity of FDG-PET is superior to CT for staging non-small cell lung carcinoma (NSCLC), with the main advantage being that FDG-PET has a high negative predictive value ( $>90 \%$ ) in the detection of mediastinal node metastasis in patients without previous chemotherapy. ${ }^{27}$ Approximately $30 \%$ of positive findings for mediastinal nodal involvement are false positives. As a consequence, if a true positive would imply a change in the therapeutic approach or intention, then any suspected pathological focus detected by FDG-PET should be confirmed by mediastinoscopy or by ultrasoundguided endoscopy with fine-needle biopsy. When FDG-PET is used for planning radiotherapy, the treatment volumes are often smaller-mediastinal volumes may be smaller in $80 \%$ of patients. This has an important benefit: the therapeutic dose can be increased with less toxicity to surrounding organs. FDG-PET has also been shown to be capable of detecting distant metastasis in more than $30 \%$ of stage III patients previously staged as M0 by conventional techniques. ${ }^{27}$ In such patients, this finding implies a change in the surgical approach and perhaps even in the treatment intention.

PET is widely used for diagnostic staging, early assessment of response to therapy, restaging, and diagnosis of recurrence in many types of gastrointestinal tumours. However, due to its low sensitivity in detecting nodal disease in esophageal cancer, radiotherapy to these areas cannot be omitted even with a negative finding on PET. On the other hand, PET has a higher specificity in ruling out nodal disease, ${ }^{28}$ so that any deposits in the supraclavicular or celiac trunk nodes can be considered positive and should be included in the GTV.

In conclusion, interpretation of the PET to select RT volumes and fields depends on the intrinsic diagnostic values for each situation and the therapeutic intention.

\subsection{Interpretation}

The first step is to visually assess the image. The physiological distribution of FDG and any variation from normal should be estimated. ${ }^{29}$ All increases in FDG uptake relative to surrounding tissue and/or equivalent regions that do not correspond with physiological uptake should be considered malignant.

Optionally, a semiquantitative study can be performed to determine the SUV indices for each observed lesion. The SUV index is the uptake of the radiopharmaceutical measured in $\mathrm{MBq} / \mathrm{ml}$ in the region of interest (ROI) and the injected dose (in $\mathrm{MBq}$ ) divided by the body weight of the patient in grams.

For most tumours, the reported SUV threshold separating benign from malignant tumours is $2.5-3 \mathrm{MBq} / \mathrm{ml}$. It is important to note that these finding come from PET studies in which attenuation mapping was performed with isotopic sources $\left({ }^{68} \mathrm{Ge}\right.$ or $\left.{ }^{137} \mathrm{Cs}\right)$, not CTAC; when CTAC is performed, uptake is significantly greater, a fact that needs to be considered when interpreting SUV.

The value of the SUV index depends primarily on biological factors and tumour histology. ${ }^{30}$ In addition, SUV assessment must include numerous factors that affect its interpretation, such as when SUV levels were measured, endogenous glucose levels, the partial volume effect (PVE), and the size and location of the ROI. ${ }^{31}$

FDG distribution is not homogeneous, with uptake varying by tissue type (e.g., uptake is higher in muscle vs. fat tissue). For this reason, the formula used to calculate SUV has been modified to account for these variations and other factors such as the patient's constitution: the modified formula allows us to correct for body surface area and muscle mass.

Despite the multitude of factors affecting SUV, their impact is less important when the purpose of SUV is to evaluate and monitor the therapeutic response. This is because therapeutic response can be measured by assessing the relative change from the initial SUV value. Nevertheless, to ensure consistency from study to study, it is imperative that we standardize SUV measurements as much as possible (e.g., the same scanner and radiopharmaceutical dose should be used to monitor ongoing changes in the disease). Criteria to standardize SUV measurement have been described in several recently published consensus documents. ${ }^{32}$

Physiological uptake of ${ }^{18} \mathrm{~F}-\mathrm{FDG}$ is observed in viable tissues, including the brain, myocardium, breast, liver, spleen, stomach, intestines, kidneys, bladder, muscle, lymphoid tissue, bone marrow, salivary glands, thymus gland, uterus, ovaries, testicles, and brown fat. For whole body studies, the sensitivity of ${ }^{18} \mathrm{~F}$-FDG to detect cerebral brain metastases is low, mostly due to the high physiological uptake of grey matter.

High uptake of ${ }^{18} \mathrm{~F}-\mathrm{FDG}$ is observed not only in neoplastic processes, but also in granulation tissue (i.e., wounds), infections, and other inflammatory processes. If we wish to interpret PET-CT images correctly, we must take these factors into consideration. In order to differentiate between benign and malignant lesions, we must consider ${ }^{18} \mathrm{~F}$-FDG uptake patterns and the specific findings of the CT in the larger context of the patient's medical history, physical exam, and other imaging techniques. In evaluating response to therapy, it is especially necessary to perform a semi-quantitative estimation (SUV).

\subsection{What is the SUV? Definition}

FDG concentration in a cancer patient can be assessed in many ways, some of which are relatively simple and others highly complex. The simplest method is a qualitative evaluation that requiring only a good quality image. In contrast, certain quantitative methods-such as the space-time quantitative description-require kinetic methods, dynamic studies and complex data analyses. Somewhere between the simplest and most complex methods lies the SUV, which is a reasonable compromise.

SUV stands for Standardized Uptake Value, a measure of the radioactivity concentration $(\mathrm{Bq} / \mathrm{ml})$ in a specific area. The 
formula to calculate the SUV is as follows:

$$
\begin{aligned}
& \text { SUV }_{\text {body weight }(\mathrm{kg} / \mathrm{ml})} \\
& =\frac{\text { decay corrected activity in region of interest }(\mathrm{Bq} / \mathrm{ml})}{\text { injected dose }(\mathrm{bq}) / \text { body weight }(\mathrm{kg})}
\end{aligned}
$$

Ideally, this formula would yield values of sufficient magnitude to function as reference values, making them independent of the particular study and patient; in such a scenario, a specific uptake value would be considered pathological if above a specific level. However, the reality is that SUV is influenced by multiple factors and is highly variable. The factors that have the largest influence on SUV are shown in Table $2 .{ }^{33}$

SUV is not a real physical value, but rather a relative value that depends on factors such as the size of the Voxel, patient movement, characteristics of the PET scanner, the technique used for image reconstruction, and the number of iterations in the reconstruction. While some factors are not under our control, others are, and to reduce variability in these factors we need to standardize measurements as much as possible. ${ }^{34}$ Guidelines are needed to standardize quantification in future FDG-PET studies; otherwise, comparison between hospitals will not be possible.

\section{Segmentation methods for metabolic images}

As discussed in the introduction, the radiation treatment plan must be based on the most precise diagnostic methods available. In 2006, an IAEA expert panel reviewed the use of PET-CT in RT planning and one of their main conclusions was the need to accurately assess disease spread before planning and delivering radiotherapy. ${ }^{7}$ In many types of tumours, PET-CT has proven to have superior specificity and sensitivity compared to CT alone, mainly as a consequence of the extra information provided by metabolic data. These findings have stimulated interest in PET-CT for both diagnosis and treatment planning.

Newer, more advanced technologies such as intensitymodulated RT (IMRT) and image-guided RT (IGRT) allow highly precise delivery of conformal doses to the tumour with steep dose gradients. At the same time, however, such steep gradients make precise contouring of the target volume more important than ever before.

The images used in radiotherapy planning allow us to define the GTV for the primary tumour and any nodal disease. Classically, CT has been the tool of choice to obtain the morphological tumour image and its main advantage is that it allows contouring directly over the tumour image. In contrast, an important disadvantage of CT-based images is that they are less useful in defining the CTV. Fortunately, the emergence of PET imaging and its ability to provide functional information is yielding new information about GTV subvolumes, and these data can be included when contouring volumes. Some authors now even use the terms "metabolic" or "biological"35 volumes based on metabolic data from the PET scan. Despite the appeal of such terms, the ICRU 83 report advises against using these terms, suggesting instead that we specify the type of study performed (GTV FDG-PET, GTV PET-FMISO, etc.) and when the image was obtained ( $0 \mathrm{~Gy}, 45 \mathrm{~Gy}$, etc.). Other specialists have suggested that PET can directly define the CTV. ${ }^{36}$ It would seem, for the moment anyhow, that the low resolution $(\sim 5 \mathrm{~mm})$ of current scanners does not allow us to make such bold claims. Nevertheless, other authors are even more optimistic, suggesting that functional and biological imaging will eventually allow us to move from a "clinical" to a "real" volume. $^{37}$

Radiation oncologists are accustomed to using the sharp, clear images provided by CT to delineate treatment volumes. The images produce by PET scans are, in contrast, much "fuzzier" and, as a result, the intra- and inter-observer variability of PET-contoured volumes are potentially much greater than volumes delineated on conventional CT images. Moreover, selection of the "window" in which treatment volumes are contoured is even more important.

Segmentation refers to the act of segmenting volumes (cutting or splitting into segments). When using a PET image to contour our GTV, we must choose the area (segment) that lies within our GTV and which, therefore, we interpret as the tumour target.

When an image is acquired and used for treatment planning, we move from the "tumour reality" to the "tumour image" and from this image we must then infer the "tumour reality". If we overestimate the real tumour volume, the result will be an unnecessary increase in the size of the treatment volume, which could lead to increased toxicity to the adjacent OARs. Underestimation, in contrast, could result in a dangerous loss of local tumour control.

Various segmentation methods have been used to plan RT treatments with PET-based images. The "gold standard" for validating these methods has generally been the pathological specimen. Nevertheless, this validation method may be less than ideal given the likelihood that "ex vivo" analysis of the sample (particularly the specimen volume) may not correspond to its "in vivo" characteristics (e.g., lung tissue).

The aim of segmentation is to classify the image in terms of "tumour or not tumour". Classification is a statistical technique applied to segmentation and several different classification methods, with varying degrees of mathematical sophistication, have been used to perform segmentation.

Segmentation of PET images is difficult due to the low spatial resolution and high level of "noise" of the images. ${ }^{38}$ To overcome these obstacles, many attempts have been made to apply mathematical methods to obtain a quantitative response that is reproducible, observer-independent, and that reflects as realistically as possible the "tumour reality". However, to date, researchers have been unable to develop a reliable method that fulfils those characteristics while also reflecting the clinical experience of different specialists and the particularities of the patient and the disease.

Three methods can be used to evaluate PET images: qualitative (i.e., visual interpretation); semi-quantitative (i.e., based on SUV); and kinetic-quantitative (i.e., a method that accounts for variation in uptake time). The segmentation methods can be divided into three categories, as follows: 
Table 2 - Most relevant factors impacting the SUV.

\begin{tabular}{|c|c|}
\hline $\mathrm{d}$ & \\
\hline $\begin{array}{l}\text { Not controllable } \\
\text { - Patient size } \\
\text { - Postural flexibility of the patient for } \\
\text { the various treatment positions. } \\
\text { - Capacity of patient to remain } \\
\text { immobile in a given position. } \\
\text { - Patient movement and breathing. } \\
\text { - Levels of blood glucose and insulin. } \\
\text { - Non-uniform uptake within the } \\
\text { tumour (necrotic centre) } \\
\text { - Diffusion in the tissue. } \\
\text { Controllable } \\
\text { - Radiopharmaceutical } \\
\text { - Isotope emission } \beta^{+} \\
\text {- Dose administered. } \\
\text { - Residual activity in the needle. } \\
\text { - Uptake time before acquisition of } \\
\text { images } \\
\text { - Acquisition time }\end{array}$ & $\begin{array}{l}\text { - Partial volume ef } \\
\text { - Discrimination in } \\
\text { - Sensitivity } \\
\text { - Mode of acquisiti } \\
\text { vs. Germod used to c } \\
\text { - Truncation } \\
\text { - Movement (differ } \\
\text { PET) } \\
\text { - Acquisition with } \\
\text { - Availability of cor } \\
\text { - Artefacts due to } \\
\text { - Use of contrast a } \\
\text { - Subtraction of th } \\
\text { - Reconstruction A } \\
\text { Projection vs. OSEI } \\
\text { - Reconstruction b } \\
\text { - Incorrect synchrc }\end{array}$ \\
\hline $\begin{array}{l}\text { 1. Visual or manual (qualitative } \\
\text { he first segmentation metho } \\
\text { gmentation-is the most intuitiv } \\
\text { dbjectivity of the method, it is no le } \\
\text { s the name implies, visual segmenta } \\
\text { tive analysis of the images; in expe } \\
\text { raluation can be quite useful. Typi } \\
\text { a visual assessment of the PET im } \\
\text { eas of pathological uptake. These a } \\
\text { CT to take advantage of the grea } \\
\text { T, which allows for a clear definitio } \\
\text { nlike PET). However, one criticism } \\
\text { athological metabolic uptake may } \\
\text { bundaries visualized on CT. }{ }^{39}\end{array}$ & $\begin{array}{l}\text { or manua } \\
\text { ite the obviou } \\
\text { than the others } \\
\text { pased on a quali } \\
\text {, this qualitativ } \\
\text { is method relie } \\
\text { entify and selec } \\
\text { then contoure } \\
\text { ial resolution o } \\
\text { tumour border } \\
\text { approach is tha } \\
\text { incide with th }\end{array}$ \\
\hline
\end{tabular}

\subsection{Threshold methods (semi-quantitative)}

The subjectivity of the visual method-with its intra- and inter-observer variability-has prompted a search for automated segmentation methods to help reduce variability. The threshold method is based on a semiquantitative analysis (i.e., SUV) of the images. To implement this methodology, however, we need to identify the multiple factors that can affect quantification of PET images (see Section 5.2 for more details).

Threshold methods use a specified uptake level (the threshold level) as a cut-off to determine whether a tissue area should be included in the treatment volume. Some threshold methods use absolute SUV cut-off values while others use a percentage of maximum SUV. Studies in phantoms ${ }^{40}$ have been performed to determine the relationship between the real volume of a lesion and the optimal threshold level that accurately depicts that real volume: in low-contrast lesions (low signal-to-background ratio [S/B]), adequate threshold levels were typically higher. This type of automated segmentation requires an a priori estimate of the size of the lesion of interest, usually obtained by CT. In tumours larger than $4 \mathrm{ml}$ and $\mathrm{S} / \mathrm{B}>5$, the size of the lesion can be predicted with a reasonable degree of accuracy when fixed threshold levels ranging from $36 \%$ to $44 \%$ of the maximum SUV are used. However, this is not the case in smaller tumours.

To solve this problem, Daisne and Gregoire ${ }^{41}$ validated a segmentation method that was independent of the size of the object of interest. They showed that selection of the proper activity threshold was actually a function of the S/B ratio, at least for volumes greater than $2 \mathrm{ml}$ with a $\mathrm{S} / \mathrm{B}$ ratio $>1.5$. They validated this method for several reconstruction algorithms.

Davis $^{36}$ later described a new concept called the "relative threshold level". This new threshold level is also a percentage of the maximum signal, but with the background signal previously subtracted (Fig. 2).

The relative threshold level that most accurately represents the real volume is $41 \pm 2.5 \%$ (background subtracted from the signal); however, it should be noted that this relative threshold overestimates the real volume in tumours $<12.5 \mathrm{~mm}$. In a letter to the editor, ${ }^{42}$ Hoffmann et al. pointed out the limitations of this method. They noted that the

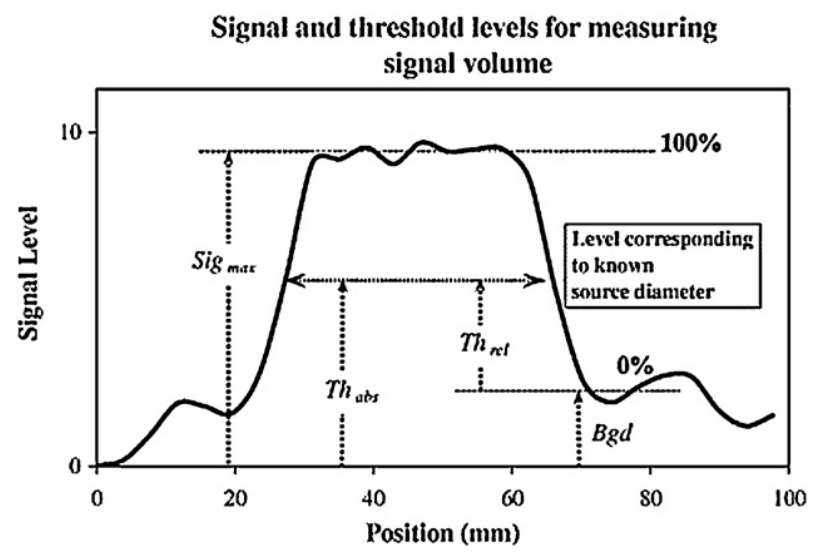

Fig. 2 - Absolute threshold = background + relative threshold (maximum signal - background).

Reprinted from Radiother Oncol 2006;80:43-50 by J.B. Davis, Assessment of ${ }^{18}$ F PET signals for target volume definition, copyright (year) with permission from Elsevier. 


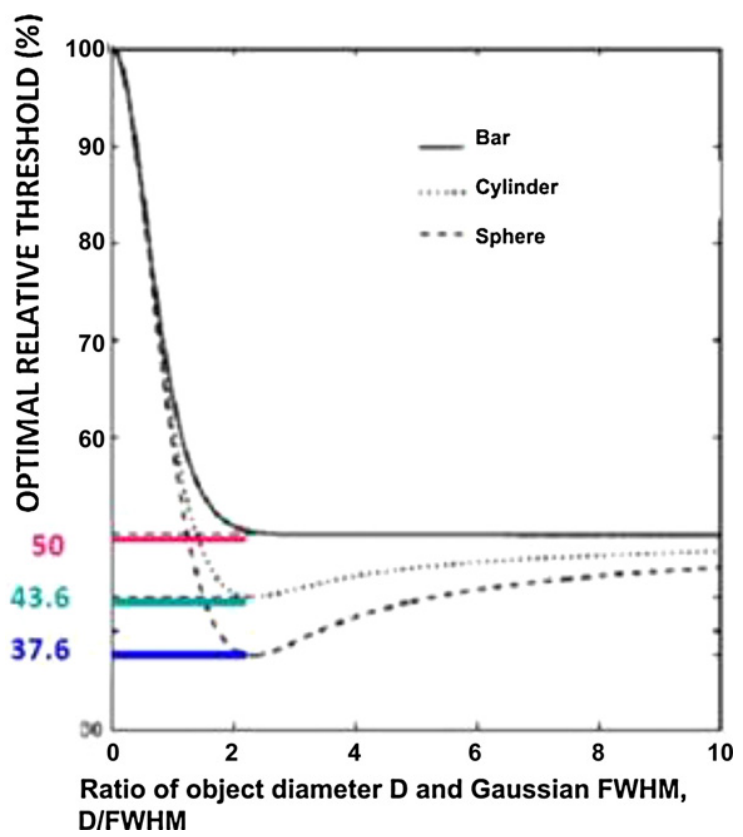

Fig. 3 - Optimal threshold value depending on the shape. Reprinted from Medical Physics (King MA, Long DT, Brill AB. SPECT volume quantitation: influence of spatial resolution, source size and shape, and voxel size. Med Phys 1991;18:1016-24) with kind permission from Springer Science and Business Media.

optimal threshold necessary to find the true "border" is a function of the diameter and shape of the tumour, as King et al. ${ }^{43}$ also pointed out (Fig. 3). As a result, there is no optimal threshold level that can be applied to the myriad sizes and shapes of tumours.

The optimal threshold depends on the size, shape, and uptake levels of the lesion (Fig. 4). Fig. 4 shows the real silhouette (in black) of a virtual phantom with an irregular shape ("peaks"), a vertical gradient of the grey value within background noise. The isocurves are delineated in red. Note that the isocurve that fits the upper peak well actually

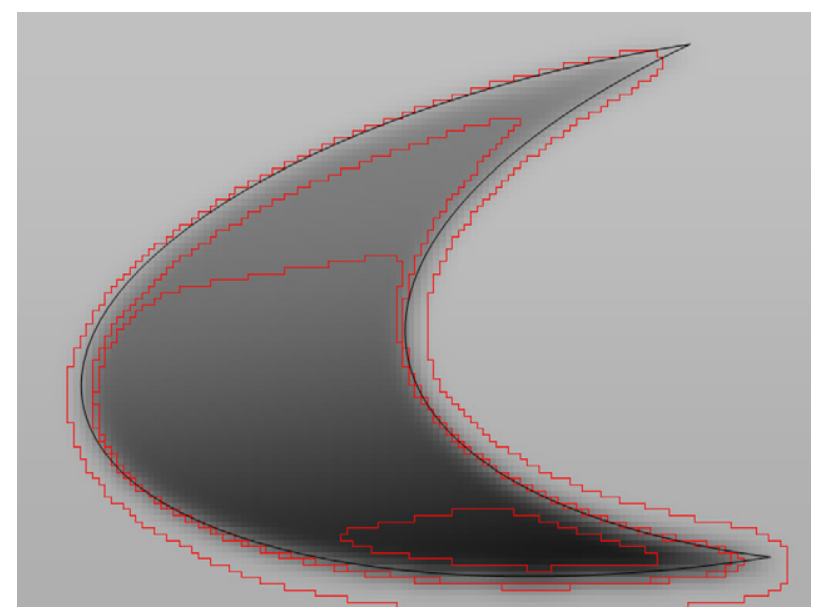

Fig. 4 - Differences in delineating a real object at different threshold levels. overestimates the lower peak. The same is true when the isocurve is optimized to fit the lower peak. In short, even when various threshold levels (red line) are used, it is not possible to reliably reproduce the original image (black).

For an object whose diameter is less than 10 times the resolution (given as full width at half maximum; FWHM), there is a single threshold value that represents the real volume only if: (1) the object is spherical; (2) uptake is uniform both within and without the object; and (3) the point-spread function (PSF) is isotropic and constant throughout the entire FOV. Unfortunately, tumours in the real world do not meet these conditions, so it will be difficult to find an optimal threshold level that precisely reflects the actual tumour.

Bielh et al. ${ }^{44}$ showed that there is a logarithmic relationship between the tumour volume and the optimal threshold level, using CT-contoured lung tumours as a gold standard to verify the relation. This inverse relation (the smaller the tumour volume, the larger the threshold needed) was subsequently proven in phantoms. ${ }^{45,46}$

In general, for small spheres, a high threshold (>50\%) compensates for uptake loss typical of the partial volume effect. For medium size spheres, the PVE is negligible but the borders are not as sharp, thus requiring a lower threshold. In large spheres, the PVE and the fuzziness of the borders are negligible and the threshold is approximately 50\%. Thus, threshold levels of $35 \%-45 \%$ correspond to radius/FWHM ratios typical of medium-sized lesions (approximately $4 \mathrm{~cm}$ diameter) represented in conventional PET (with a FWHM of 4-7 mm), a fact that supports optimal threshold levels of approximately 40\%-precisely the levels recommended by numerous authors.

The proper threshold to reflect the correct size of a lesion depends both on the signal-to-noise ratio as well as the PVE. To determine this relationship, we must first obtain a calibration curve in phantoms with spheres of a known size (Fig. 5) and the same radioactivity.

Prior calibration is essential in order to determine the correct threshold level that should be applied to a lesion of a given size once the signal-to-noise ratio of the specific PET-CT scanner is known. Also, by calibrating the scanner, we can later perform comparisons between centres.

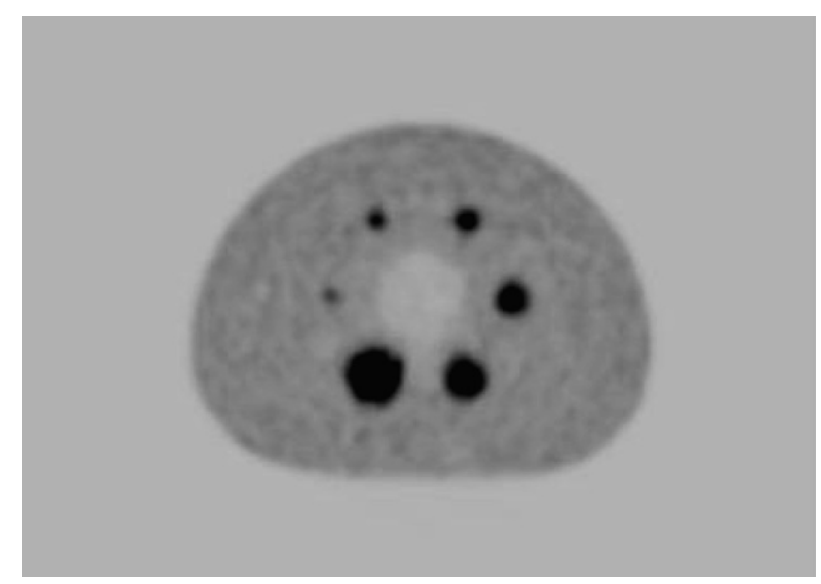

Fig. 5 - Partial volume effect. 


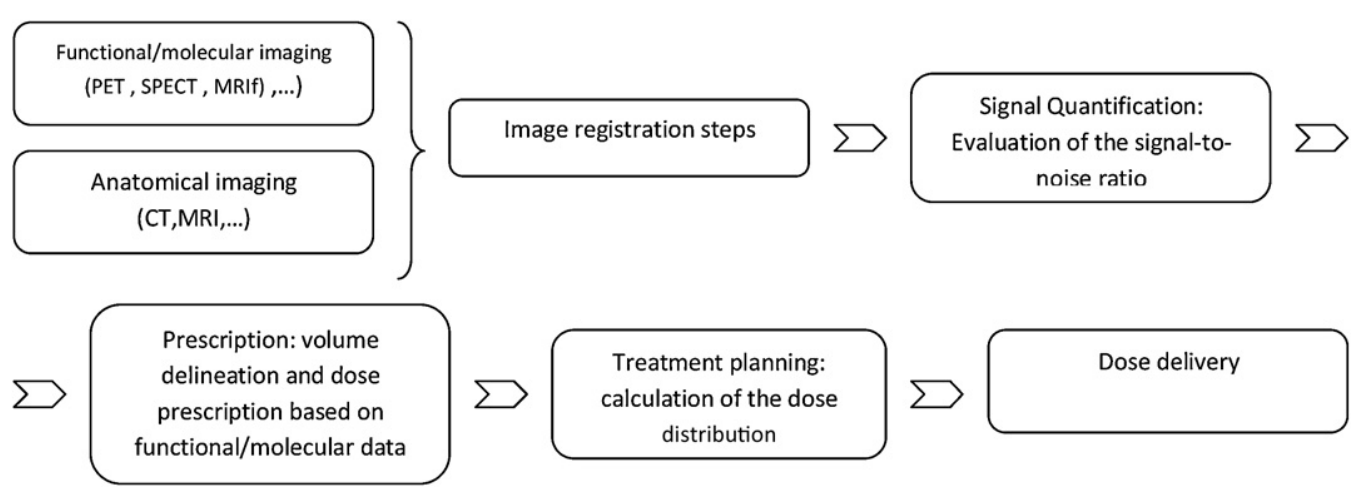

Fig. 6 - Definition of the registration and fusion processes.

\subsection{Variational approaches (gradient method)}

The aim of these methods is to assess variation in intensity to identify the maximum gradient of intensity between tumour uptake and background uptake. The point or area of maximum variation is assumed to represent the lesion borders.

The "gradient" method was first described by Geets and colleagues in 2007. ${ }^{47}$ Using pathological specimens from head \& neck cancer patients as a gold standard for validation, this method reduced the size of the false positive volume but at the cost of increasing the false negative volume. In other words, the gradient method reduced overestimates of the real volume but increased the risk of missing part of the tumour. Subsequently, other gradient methods, available commercially and validated pathologically in lung tumours, have been described. ${ }^{48}$ One of the main limitations of gradient methods is their high sensitivity to background noise. ${ }^{49}$

\subsection{Other methods}

Due to their mathematical complexity, anyone wishing to use the highly sophisticated methods mentioned below will need to consult a specialist with real expertise in these techniques. These models use complex algorithms to compensate for the deficiencies of the previously described methods. Importantly, however, the difference in results between these highly complex methods and the other methods described above is only a few millimetres. The subpixel precision of some of these methods is such that is of little value in current daily clinical practice.

Tumour size and heterogeneity have less of an impact on these methods, but image quality needs to be excellent. Two of the more common of these methods are learning methods and stochastics. Segmentation methods based on a kineticquantitative analysis are still being studied and none are yet available for clinical use.

To conclude this section, we can state that the most robust and best method has yet to be determined. However, to identify the best method, we will first need to standardize our approaches and then compare findings among different groups. In general, we advise against the use of fixed thresholds; instead, we recommend the use of adaptive thresholds that take background noise into account. Keep in mind, however, that flexible thresholds require precise calibration.
Finally, the extreme complexity of some methods (stochastics, learning methods, etc.) is probably not worth the effort, at least at present. Frankly, for departments without strong support from the medical physics department, we recommend manual segmentation. ${ }^{38}$

\section{Physics and radioprotection}

\subsection{Why quality control is needed}

The addition of functional imaging to radiotherapy planning requires the inclusion of biological information (in addition to anatomical information obtained mainly through CT and MRI) to provide information about the functional and molecular heterogeneity of the target tissues and in some cases, the adjacent healthy tissues (Fig. 6).

The use of multimodal imaging, particularly functional imaging, implies a change in the radiotherapy process, as two new steps (image registration and signal quantification) must be added. The first step, image registration, is performed to "geographically" determine the biological information so that a one-to-one correspondence between the anatomical and functional pixels can be made. The second step, signal quantification, is performed prior to volume delineation to establish the qualitative (if referring to a specific molecule) or quantitative (if referring to signal intensity) signal level that indicates the existence of a target volume. Performing these two steps will then allow us to continue with the usual radiotherapy planning and treatment process. In the present section we discuss some aspects of these steps. However, for detailed, step-by-step quality control protocol (frequency, etc.), we encourage readers to refer to the references cited.

\subsection{Quality control of the equipment}

The objective of quality control of the PET-CT system is to verify the operational integrity of the detectors and electronic chain used for image acquisition in order to maintain a high-quality image while minimizing artefacts. To incorporate PET-CT images for radiotherapy planning, quantification and registration must be precise, which is why quality control checks need to be performed. The typical CT scanner quality 


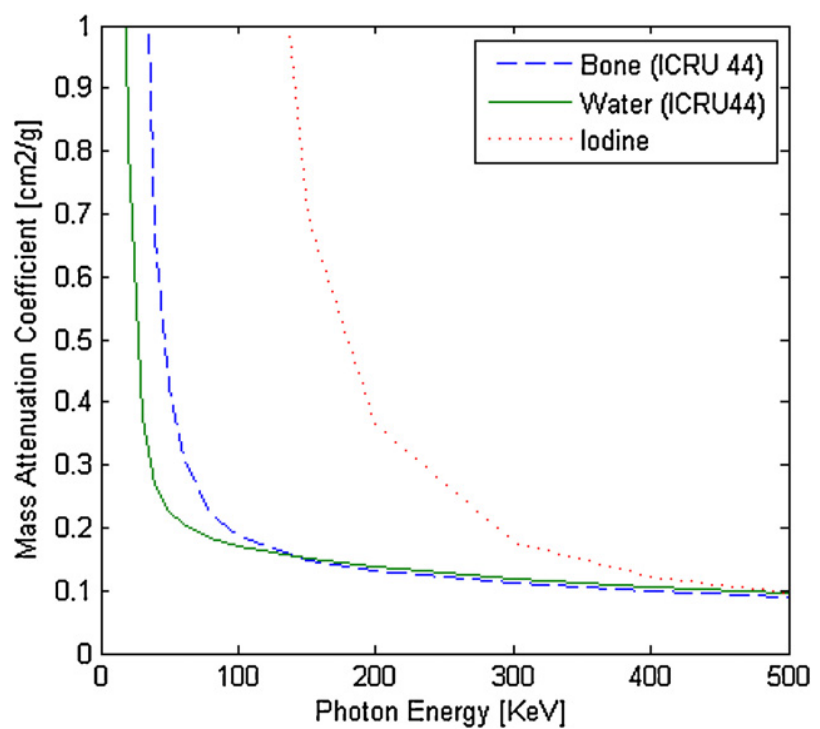

Fig. 7 - Dependence of the linear attenuation coefficient on photon energy. Figure created from data published by Hubbell and Seltzer ${ }^{57}$.

control tests used in RT, such as those listed below, should be performed. ${ }^{50-53}$

- Verification of laser centering system.

- Precision in the table top movements.

- Image quality.

- Patient dose registration (CT dose index).

- Transfer and manipulation of the image in the TPS.

In addition to quality control of the CT scanner, we must also check the PET. For instance, we must consider plane alignment between CT and PET imaging studies, and also new functions related to registration, fusion, segmentation, and influence of the dose calibrator. ${ }^{54,55}$

In the following section we describe the essential quality control tests and procedures for SUV-based PET-CT imaging in radiotherapy treatment planning. ${ }^{54}$ In a perfect world, each of these tests would produce a numerical value (an uncertainty factor) that would permit us to precisely calculate the additional margin needed to incorporate that uncertainty into our clinical practice; the resulting margin could then be used to define the treatment volumes. These tests still provide useful information, but mostly to establish tolerance ranges that indicate whether the treatment is acceptable or not. The tests selected will depend on the degree of precision desired: a qualitative evaluation of the diagnosis requires less precision than a quantitative description of changes in uptake. This decision will determine the most relevant tests needed for both quality control procedures and tolerances.

\subsubsection{Acquisition equipment}

7.2.1.1. Mechanical tests. In this section we have included tests designed to assure the integrity of the coordinates system, defined by laser alignment and agreement between the coordinates system, and that of the PET and CT imaging planes. Most of the latest scanners include tools to perform end-to-end testing to assure that the CT and PET planes coincide.

The main tests should verify the following. Laser alignment:

- Alignment between the gantry and room lasers. Both systems should not only be coincident, but parallel.

- Alignment between the gantry laser and the imaging plane from the coordinate origin of the imaging study.

- The laser projections and the scanner should be parallel and orthogonal with respect to the imaging planes.

Table top movement:

- Orthogonal movement relative to the image acquisition plane.

- Precision and reproducibility of both longitudinal and vertical movements.

- Verification of the precision of the table top indexing and positioning, controlled by the scanner.

- Verification of the previous indication under clinical conditions with a weighted table.

A flat, indexable table top should be used together with the immobilization devices in order to exactly reproduce the conditions under which both simulation and treatment are carried out.

PET-CT alignment:

- Multimodality phantoms should be used for end-to-end testing (CT/MRI/PET-CT). The object of the test is to verify correct registration of the reconstructed CT and PET images. To do this, a phantom that allows radioisotope-filled ("hotrod") inserts inside or outside the phantom should be used to verify agreement between the PET and CT images under clinical conditions. Usually, the manufacturer can provide such a phantom, often composed of ${ }^{68} \mathrm{Ge}$ rods, for use in calibration and correction of any offset between the PET and CT planes.

7.2.1.2. Quality control tests for the images. In general, these tests are designed to assess and assure the reliability of image quality.

- Homogeneity and lack of artefacts. Both modalities assure topographic uniformity. In reconstruction of the PET image, the CT transmission image is used to perform attenuation correction, due to the different distances travelled by the photons emitted from the interior and surface of the phantom and due to differences in energy spectrum between CT and PET. Attenuation correction can produce artefacts when contrast agents are used, or in patients with a prosthesis. ${ }^{56}$

- Resolution and contrast of CT and PET images. PET resolution is measured in and outside of the axis and this directly affects signal quantification (see below for a more in-depth discussion of this aspect).

- Determination of the attenuation coefficients for various electron densities. Attenuation coefficients are energy dependent and attenuation also depends on the material-soft tissue, bone, and iodine (used in most 


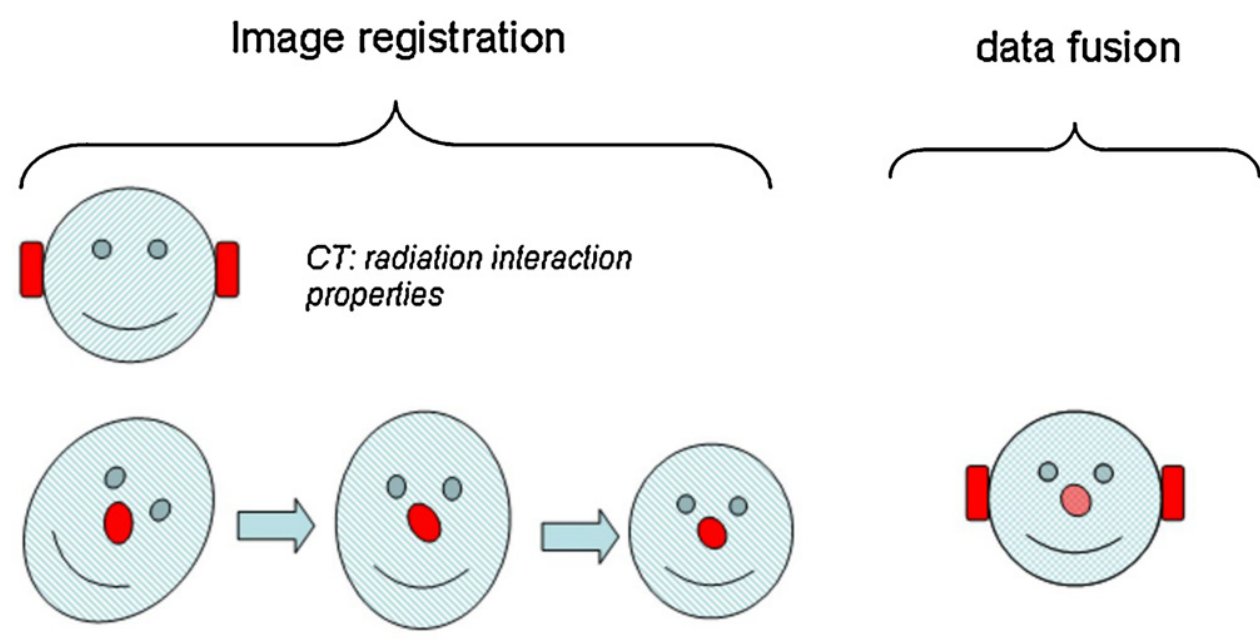

Fig. 8 - Definition of the registration and fusion processes.

contrast agents). To some extent, electron densities depend on the kilovoltage used (Fig. 7)..$^{56}$

\section{Quantification tests of the PET signal:}

- Sensitivity. 2D and 3D calibration. The purpose is to determine the rate of coincidence events per unit of radioactivity concentration. This factor is then used to calculate the image pixel value, given in concentration units.

- Quantification of the PVE. The partial volume effect is due to the limited spatial resolution of the detectors. This results in a signal loss in the tissues of a size that approaches the resolution limits of the imaging system. This signal loss can be quantified in phantoms through the use of hot-rod inserts of

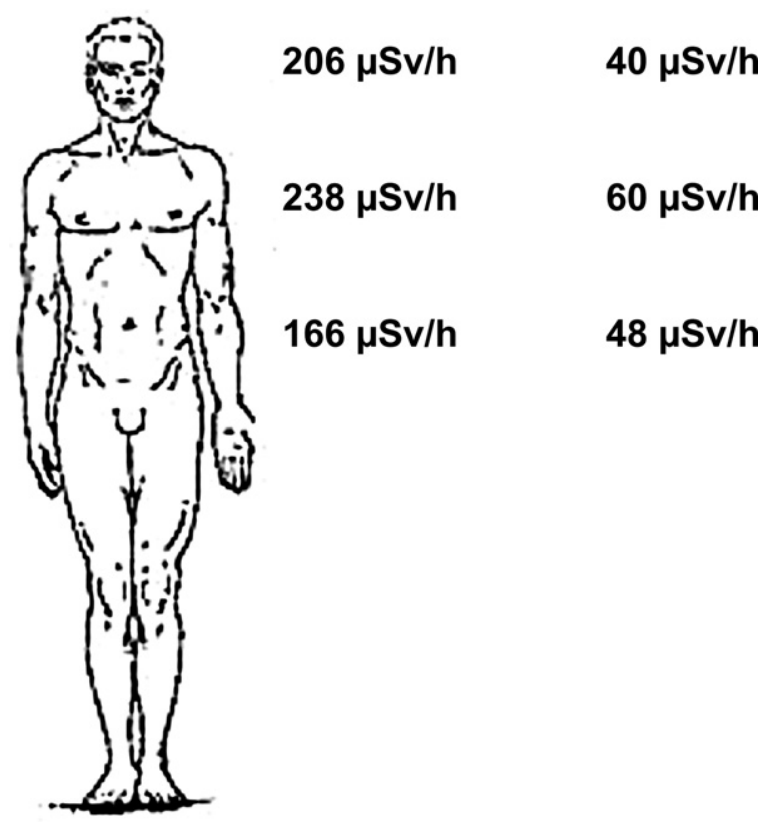

Fig. 9 - Dose rate at $10 \mathrm{~cm}$ and $1 \mathrm{~m}$ on average for 10 patients injected with approximately $370 \mathrm{MBq}$ and at $60 \mathrm{~min}$ following injection. varying sizes under various conditions (dependence on the tracer concentration, radioactive background, movement, etc.) by which various curves are obtained under different conditions that are applied on segmentation. ${ }^{41,58,59}$ This effect can also be affected by the type of reconstruction algorithm used (Filtered Back Projection, OSEM, etc.)

\subsubsection{Quality control of the}

registration/fusion/segmentation software

Segmentation of the volumes of interest can be performed with a dedicated workstation designed for this purpose (the Contouring Workstation). Alternatively, the radiotherapy TPS can also be used. The objective of the quality control tests in this section is to assure that the radiation oncologist has received the correct data needed to prescribe treatment and reliably delineate the targets. Data integrity and spatial integrity should both be verified:

- Data transfer. The systems used to transfer data must be checked for compatibility to ensure that all data from the copied file is transferred completely, either to the hard disk or to the DICOM transfer nodes of the hospital network. Check that the information from the DICOM attributes and the digital matrix of the image are both maintained so that there is no loss of data due to conversion. Verification should include both series of images (from the CT and PET). When purchasing new systems, it is advisable to demand compatibility between the PET-CT scanner and the TPS. Connectivity between two pieces of equipment can be evaluated by the use of the equipment's DICOM Conformance Statements. This is relevant to determine what type of information is transmitted, especially when absolute quantification of the signal is important.

- Image registration. This process consists of overlaying 2 or more images of the same scene either acquired at different times and/or from different angles, and, frequently, with different sensors. Before fusion can be performed, the pixels from all images must be matched one-to-one. This involves a series of transformations: turns, scaling, and, at times, deformations (Fig. 8). 
In hybrid PET-CT scanners, the hardware is used to perform registration. In each imaging modality, registration is based on the relative movement of the patient to the detectors within a known range. In an ideal world, image registration would be perfectly precise if the following conditions were true: the patient remains completely immobile; the table top is not deformed by weight and is moved precisely between the coordinates defined for each modality; and the CT and PET imaging planes are exactly parallel to each other and perpendicular to the table top. Unfortunately, this is not the case in actual practice. For this reason, the purpose of verification testing is to evaluate the degree of misalignment to determine its effect on the geometric margins that need to be applied to the contoured PET volumes. Quantification of this discrepancy should be considered a systematic error. The aim of this type of test is similar to that described above for the end-to-end test (i.e., to verify alignment between the CT and PET) except that, in this case, we evaluate the images on the Contouring Workstation. To simulate the patient load, we must add weight-the IAEA recommends adding $100 \mathrm{~kg}$ - to the table top. ${ }^{54}$

Accuracy of the fusion algorithms. Fusion involves synthesizing the data provided by the different images into a single set of data. Fusion is not exclusive to PET-CT imaging, as it is performed in all imaging modalities (MRI and ultrasound, among others). Due to qualitative differences in the data, registration is more complicated when different types of images are co-registered. A rigid phantom is used to quantify the error generated by the fusion algorithms (mutual information, etc.). In deformable geometries, quantification is difficult due to the lack of simple analytical tools and also because all deformable registration methods have a limited range of applicability, either because they were designed only for specific cases (e.g., 4D) or due to a lack of a consistent physical model (e.g., deformation of bone tissue as if it were soft tissue). In all cases, these tests should be performed by a well-trained expert to avoid generating a larger error than the one being corrected.

Precision of segmentation tools. Automated segmentation tools (by threshold, gradient, etc.) for the PET image should also be checked to verify that these perform exactly as expected. For example, these tools should not introduce any information not contained in the original image (via softening or enhancement filters, for instance) and there should be no hidden signal correctors. Likewise, check to be sure that there is no loss of the absolute signal due to normalization in percentages. To counter the PVE, approximate mathematical methods can be used, although these must first be verified with simple geometric figures (following manufacturer specifications). Note that if these verification tools are not available in the software package, the dimensions of small or elongated volumes may be underestimated. It is important to verify that the tumour image identified by the automated segmentation process can be exported to a DICOM format that is compatible with the TPS used in RT planning. Although workstations in the Department of Nuclear Medicine allow for definition of the ROI based on a predetermined minimum level of intensity, the resulting image is, unfortunately, not compatible with the software used in Radiation Oncology.

Accuracy of the quantification tools. The system should be capable of providing quantitative information from both the CT and the PET. This includes statistical data about the volumes, ROIs (mean, deviation, histograms, etc.) as well as the pixel values in Hounsfield units (the most common) and radioactivity concentration $(\mathrm{kBq} / \mathrm{ml})$ and, if possible, for SUV uptake. As described in the previous section, if the uptake volumes are too small, the PVE will distort SUV values; the impact of PVE is thus particularly relevant for small volumes. Verification should be performed with known patterns, either by calibrated sources or tracer quantities that have been calibrated (by in-house dose calibrators) in phantoms with known geometries and different ranges of background activity. In all cases, the traceability of the measuring chain should be verified. ${ }^{41}$

Archive and security copies. Verify that archiving and copying actions are performed accurately and that it is possible to recover data from a historical patient in a reasonable amount of time without undue difficulty. For permanent storage in a PACS, assure compatibility of the PACS and the storage integrity of the digital data.

\subsubsection{Quality control for the treatment planning system}

Verify that the TPS includes the density table for the CT. This seems obvious, but occasionally it is overlooked because PET-CT units are often located in the Nuclear Medicine Department.

DICOM-RT. RT structure set. If transferring images with volumes that have been delineated on an external station, verify that the volume has been transferred correctly.

\subsubsection{Radiopharmaceutical dispensing unit/dose} calibrator

Calibration: obtaining the calibration factor for the dose calibrator. Because tools to measure radioactivity concentration may not always be available, doses are sometimes dispensed by volume. However, this can lead to large errors in dispensation. Any calibration errors will be transferred to the PET unit and will therefore affect the $\mathrm{kBq} / \mathrm{ml}$ of the pixel value if this is used to determine the counts rate/activity (see sensitivity).

Detector stability test. Verify stability with a Cs-137 source at the start of the day and check contamination throughout the day (especially important when the scanner is used on two consecutive both morning and afternoon).

\subsubsection{Routine and periodic testing of the PET and CT}

We used the data provided by Refs. 54 and 55, which the reader is encouraged to consult for more details, to create Tables 3 and 4, which describe the other quality control tests-and the frequency thereof-that should be performed for PET-CT scanners. These recommendations are general, and not specific to radiotherapy. Finally, we also recommend that the reader consult the manufacturer's PET-CT manual, which can be used to adapt these tests to their particular situation (i.e., based on available resources and scanner characteristics).

\subsection{Radiological protection for patients and workers}

It is important to assess the radiation level in the PET-CT room and the dose received by the nuclear medicine and radiotherapy technologists as well as the patients. 
Table 3 - Routine tests and frequency thereof for the PET scanner.

PET

\begin{tabular}{|c|c|c|c|}
\hline Daily & Weekly & Quarterly & Annual \\
\hline $\begin{array}{l}\text { Detector stability check. } \\
\text { Inspect sinograms or } \\
\text { compare to a pattern. } \\
\text { Gain normalization }\end{array}$ & $\begin{array}{l}\text { Quantification scan } \\
\text { Compare ROI to } \\
\text { calibrated radioactivity }\end{array}$ & $\begin{array}{l}\text { Calibration of photomultipliers } \\
\text { Coincidence Timing Resolution } \\
\text { Time of flight } \\
\text { Normalization } \\
\text { Cross-calibration with the } \\
\text { well-counter }\end{array}$ & $\begin{array}{l}\text { Uniformity } \\
\text { Resolution } \\
\text { Count rate } \\
\text { Correction of dead time } \\
\text { losses } \\
\text { Sensitivity } \\
\text { Quantification } \\
\text { Table movement }\end{array}$ \\
\hline
\end{tabular}

\subsubsection{ALARA criteria}

ALARA ("as low as reasonably achievable") refers to the general aim of minimizing the radiation dose to workers and patients. The RT technologist should be aware that patients undergoing planning PET-CT have been injected with a radionuclide and are, therefore, a source of external radiation and contamination. To reduce exposure time, it is essential that technologists prepare thoroughly and carefully before coming into contact with the patient. The use of shoes, gloves, and disposable aprons all help to minimize contact-related contamination.

Following ALARA criteria, all immobilization devices, including thermoplastic or vacuum-moulded devices, should be prepared before radiopharmaceutical administration. The patient can be repositioned in the PET-CT unit after injection. Because it takes longer to reposition and align a previously tattooed patient after radiopharmaceutical injection than it does to place the radiopaque markers and mark their position, it may be preferable to apply the definitive tattoo after completion of the imaging study, when radioactivity has diminished.

The nuclear medicine technologist should be aware that the patient will receive RT treatment based on the geometry and the coordinate origins established during image acquisition, and he or she should be familiar with the language and techniques used in this process.

There is a very real possibility of contamination during the imaging study (e.g., through vomiting or urine loss) and all staff should be trained and prepared for such events in order to contain the contamination. Appropriate decontamination material should be on hand, including contamination detectors. If the equipment becomes contaminated, decontamination may be needed before any other imaging studies can be performed.

Table 4 - General tests and frequency for CT.

CT

\begin{tabular}{lll}
\hline Daily & \multicolumn{1}{c}{ Weekly } & \multicolumn{1}{c}{ Annual } \\
\hline Water density & Calibration of & Position of slice \\
Artefacts & Hounsfield & Slice thickness \\
Uniformity & numbers & Dosimetry CTDI \\
Linearity of & Laser alignment & Resolution \\
attenuation & & Low-contrast resolution \\
coefficients & & Dosimetry CTDI \\
Low-contrast & & Table warping \\
resolution & & \\
\hline
\end{tabular}

The use of protective clothing is not effective against energy annihilation photons ( $511 \mathrm{keV}$ ) because the thickness of the lead typically used in this material is insufficient (up to $90 \%$ of photons penetrate the material).

The use of comprehensive checklists before and during the PET-CT scan can reduce exposure time and minimize the likelihood of errors, which will also reduce the probability of having to repeat the scan. In addition, clear lines of communication between the Radiation Oncology and Nuclear Medicine departments should be established to avoid errors in transmitting information that could lead to improper administration of the radiopharmaceutical.

\subsubsection{Pregnancy and lactation}

The possibility that female patients of child-bearing age might be pregnant must be considered. The risks and benefits of the scan should be thoroughly discussed with pregnant patients. Patients who are breast-feeding should be instructed to interrupt feeding for at least $18.5 \mathrm{~h}$ (10 half-lives of decay). Most of the radiation dose to the baby is caused by external radiation rather than ingestion through breast-feeding. ${ }^{56}$ It is the responsibility of the physician to explain this information to the patient before she signs the informed consent form.

\subsubsection{Children}

Due to their relatively small size, children may absorb more radiation from the CT scan than from the radiopharmaceutical. For this reason, it is important to develop protocols for image acquisition that minimize the CT dose without compromising image quality.

\subsubsection{Dose to workers}

All staff involved in handling patients should be provided with lapel, wrist, and ring dosimeters if necessary. Pregnant workers should have an abdominal dosimeter to insure that the dose to the foetus does not exceed the annual dose limit to the public ( $1 \mathrm{mSv})$.

Fig. 9 shows a sample of doses measured with a 0.51 camera at $10 \mathrm{~cm}$ and $1 \mathrm{~m}$ at the Hospital Universitario La Paz (Spain). Values are also shown for dosimeters located in the pocket of the RT technologist during patient immobilization on the PET table; patient dose is about $7 \mu \mathrm{Sv} /$ patient in this same hospital.

To obtain reference values, it is important to quantify the dose received by patients as a function of the acquisition technique and the radioactivity of the radiopharmaceutical. ${ }^{34}$ Fig. 10 shows the typical dose in different phases during FDG 


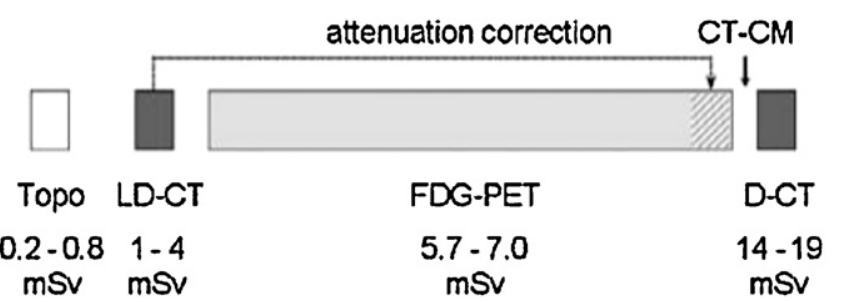

Fig. 10 - Dose received by the patient at various stages during a PET/CT imaging study with contrast media.

contrast-enhanced PET/CT, with a topogram, low dose CT, PET, and intravenous contrast CT.

\subsection{Staffing needs}

The medical physicist is responsible for carrying out the tests described above and this should be indicated in the Radiotherapy Quality Assurance Program. However, the relevant scientific societies need to collaborate to establish standardized tests.

\section{Psychological impact and patient safety}

\subsection{Psychological impact}

The gantry aperture of the PET-CT simulator is generally smaller than that of the CT-simulator. PET image acquisition also requires more time. These two factors make PET-CT more uncomfortable for the patient. Greater discomfort, in turn, implies a higher risk of movement, more muscle tension, agitated breathing, etc. Another potential cause of discomfort is the fact that patients must disrobe completely; for this reason, every effort should be made to minimize patient nudity. These and other methods designed to reduce the psychological impact on patients deserve serious consideration.

An important component of patient preparation involves providing sufficient information about the procedure before it begins. The main points that should be explained are the following:

- What a PET/CT is and how it works.

- Why PET-CT-Simulation is required.

- The approximate duration of the procedure.

- How to prepare for the procedure: fasting, medications, rectum and bladder status, exercise, dental prostheses, etc.

- The size of the gantry aperture of the PET-CT.

- The position that the patient must maintain.

- The immobilization devices to be used; if thermoplastics will be used, explain their use.

- Where and why tattoos and/or marks will be made.

- How to breathe in a relaxed and regular manner.

- Muscle relaxation and thought distraction techniques that can be used before and during the procedure.

It would be helpful to create a brochure that contains all of this important information.

\subsection{Patient safety}

During a diagnostic PET-CT scan, patient-operator communication via interphone is quite easy. However, when immobilization devices are used (as in planning PET-CT) — particularly head and neck devices such as the thermoplastic masks or bite blocks-communication may be difficult. Moreover, head \& neck patients often have compromised phonation caused by surgery or by the tumour itself. Consequently, to reduce patient anxiety and guarantee a quick response to any unexpected problems, it is essential that the patient have easy access to an emergency button or that he/she be given clear instructions to raise a hand if any problems arise. Likewise, to assure that the patient is monitored throughout the entire procedure, the availability and use of a closed-circuit television, such as those used in RT treatment rooms, is recommended. 60

Special attention should be paid to assuring permeability of the IV cannula used for contrast infusion. Similarly, the infusion pump should be checked to make certain that it is working properly. Extravasation or inadequate infusion could compromise the quality of the CT images or cause patient movement due to pain at the IV insertion point; in both of these scenarios, the scan would have to be repeated. For this reason, a test bolus with saline solution (a feature of all modern contrast injectors) should be administered. Before the IV contrast is administered, it is important to check the patient's medical record for allergies to contrast agents, proper kidney function (serum creatinine levels), and nephrotoxicity risk factors (e.g., diabetic or hypertensive nephropathy, nephrectomy, recent administration of IV contrast agents or nephrotoxic drugs).

Although unexpected allergic reactions to contrast agents are uncommon, the limited ability of immobilized patients to communicate easily makes continuous monitoring of the patient essential. It is therefore especially important to observe skin colouration and respiratory rate, and to watch for unexpected movements of the extremities or gestures that could be interpreted as signs of anxiety, discomfort, or suffering (e.g., grabbing the table, agitation, hitting with hands or feet, etc.).

\section{Conclusions}

The true utility of PET-CT in the RT planning process is still unclear. Standardization of the technique will allow us to compare clinical results and approaches from different centres before final validation.

For hospitals who wish to implement planning PET-CT for $\mathrm{RT}$, it is essential to first develop-through interdepartmental collaboration-a well-defined protocol. Similarly, it is essential to verify that all equipment needed to carry out the imaging study is available. All equipment used in the RT planning process should be checked and "tuned-up" to assure high quality and reliable diagnostic images and to offer the maximum radiological safety to the patient and staff.

To date, no ideal segmentation method has been established. Consequently, each clinical group must select the method they consider to be the most reliable and with which they are most familiar. Regardless of the segmentation 
method chosen, this information should be recorded together with details regarding the structures that were included in the final treatment volume. This information, combined with the data obtained during patient follow up, is essential in order to definitively establish the role of metabolic imaging in radiotherapy treatment planning.

\section{Conflict of interest}

None declared.

\section{Financial disclosure}

None declared.

\section{Acknowledgments}

We wish to thank Bradley Londres for translating the text from Spanish and for helping to improve the English version. In addition, we would like to express our gratitude to the current and past presidents, respectively, of the SEOR (Alfredo Ramos Aguerri, Ferran Guedea), SEFM (Juan José Peña Bernal, Natividad Ferrer García), and SEMNiM (Ángel Soriano, Francesca Pons) for their strong support for this project.

Finally, we express our gratitude to Antonio Rodríguez Fernández, president of the SEMNiM PET group, for his invaluable contributions.

\section{REFERENCES}

1. Report 83. J ICRU 2010;10(1), http://dx.doi.org/10.1093/jicru/ndq009. Oxford University Press.

2. Prescribing, recording and reporting photon beam therapy (supplement to ICRU report 50). ICRU report 62.

3. Malicki J. The importance of accurate treatment planning, delivery, and dose verification. Rep Pract Onco Radiother 2012;17(March (2)):63-5.

4. Facey K, Bradbury I, Laking G, Payne E. Overview of the clinical effectiveness of positron emission tomography imaging in selected cancers. Health Technol Assess 2007;11(October (44)), iii-iv, xi-267.

5. Delbeke D, Schöder H, Martin WH, Wahl RL. Hybrid imaging (SPECT/CT and PET/CT): improving therapeutic decisions. Semin Nucl Med 2009;39(September (5)):308-40.

6. MUFACE. Guía PET-CT. Protocolo de Prescripción. Madrid: Mutualidad General de Funcionarios Civiles del Estado; 2011 www.muface.es

7. MacManus M, Nestle U, Rosenzweig KE, et al. Use of PET and PET/CT for radiation therapy planning: IAEA expert report 2006-2007. Radiother Oncol 2009;91(April (1)):85-94.

8. The role of PET/CT in radiation treatment planning for cancer patient treatment. In: IAEA, VIENNA. 2008. IAEA-TECDOC-1603.

9. PET in radiotherapy planning. Radiother Oncol 2010;96(September (3)):275-356.

10. Mutic S. Patient positioning, immobilization devices, and fiducial markers in positron emission tomography-computed tomography scanner-based radiation therapy simulation. Semin Ultrasound CT MR 2010;31(December (6)):462-7.
11. Utilidad de la PET-TAC en Oncología. Arán ediciones; 2010, ISBN 978-84-92977-14-7. Madrid.

12. Nestle U, Kremp S, Grosu A-L. Practical integration of $\left[{ }^{18} \mathrm{~F}\right]-\mathrm{FDG}-\mathrm{PET}$ and PET-CT in the planning of radiotherapy for non-small cell lung cancer (NSCLC): the technical basis. ICRU-target volumes, problems, perspectives. Radiother Oncol 2006;81(November (2)):209-25.

13. van Dalen JA, Vogel WV, Corstens FHM, Oyen WJG. Multi-modality nuclear medicine imaging: artefacts, pitfalls and recommendations. Cancer Imaging 2007;7(May (1)):77-83.

14. García Garzón JR, Rodríguez A, Cabrera A. Tomografía por emisión de positrones de cuerpo completo (PET/TAC) con ${ }^{18}$ F-fluorodesoxiglucosa. Rev Esp Med Nucl 2009;28:85-9.

15. SERAM recommendations. Available online at: http://seram.es/ index.php?option=com_content\&view=article\&id=838\&Itemid $=204$ [accessed 09.09.12].

16. Otero HJ, Yap JT, Patak MA, et al. Evaluation of low-density neutral oral contrast material in PET/CT for tumor imaging: results of a randomized clinical trial. AJR Am J Roentgenol 2009;193(August (2)):326-32.

17. Dizendorf E, Hany TF, Buck A, von Schulthess GK, Burger C. Cause and magnitude of the error induced by oral CT contrast agent in CT-based attenuation correction of PET emission studies. J Nucl Med 2003;44(May (5)):732-8.

18. Bannas P, Weber C, Adam G, et al. Contrast-enhanced [(18)F]fluorodeoxyglucose-positron emission tomography/computed tomography for staging and radiotherapy planning in patients with anal cancer. Int $J$ Radiat Oncol Biol Phys 2011;81(October (2)):445-51.

19. Boellaard R, O'Doherty MJ, Weber WA, et al. FDG PET and PET/CT: EANM procedure guidelines for tumour PET imaging: version 1.0. Eur J Nucl Med Mol Imaging 2010;37(January (1)):181-200.

20. Delbeke D, Coleman RE, Guiberteau MJ, et al. Procedure guideline for tumor imaging with ${ }^{18}$ F-FDG PET/CT 1.0. J Nucl Med 2006;47(May (5)):885-95.

21. Coffey M, Vaandering A. Patient setup for PET/CT acquisition in radiotherapy planning. Radiother Oncol 2010;96(September (3)):298-301.

22. Sattler B, Lee JA, Lonsdale M, Coche E. PET/CT (and CT) instrumentation, image reconstruction and data transfer for radiotherapy planning. Radiother Oncol 2010;96(September (3)):288-97.

23. Leide-Svegborn $\mathrm{S}$. Radiation exposure of patients and personnel from a PET/CT procedure with ${ }^{18}$ F-FDG. Radiat Prot Dosim 2010;139(1-3):208-13.

24. Pujades Claumarchirant MC, Martí Vidal JF, Olivas Arroyo C, Bello Arqués P, Mateo Navarro A. Application of ICRP-103 in the calculation of effective dose associated to nuclear medicine tests. Rev Esp Med Nucl 2010;29(June (3)):114-21.

25. Kyzas PA, Evangelou E, Denaxa-Kyza D, Ioannidis JPA. ${ }^{18} \mathrm{~F}$-fluorodeoxyglucose positron emission tomography to evaluate cervical node metastases in patients with head and neck squamous cell carcinoma: a meta-analysis. J Natl Cancer Inst 2008;100(May (10)):712-20.

26. Troost EGC, Schinagl DAX, Bussink J, Oyen WJG, Kaanders JHAM. Clinical evidence on PET-CT for radiation therapy planning in head and neck tumours. Radiother Oncol 2010;96(September (3)):328-34.

27. Hellwig D, Baum RP, Kirsch C. FDG-PET, PET/CT and conventional nuclear medicine procedures in the evaluation of lung cancer: a systematic review. Nuklearmedizin 2009;48(2):59-69, quiz N8-9.

28. Shimizu S, Hosokawa M, Itoh K, Fujita M, Takahashi H, Shirato H. Can hybrid FDG-PET/CT detect subclinical lymph node metastasis of esophageal cancer appropriately and contribute to radiation treatment planning? A comparison of image-based and pathological findings. Int J Clin Oncol 2009;14(October (5)):421-5. 
29. Cook GJ, Fogelman I, Maisey MN. Normal physiological and benign pathological variants of 18-fluoro-2-deoxyglucose positron-emission tomography scanning: potential for error in interpretation. Semin Nucl Med 1996;26(October (4)):308-14.

30. Keyes Jr JW. SUV: standard uptake or silly useless value? J Nucl Med 1995;36(October (10)):1836-9.

31. Weber WA. Quantitative analysis of PET studies. Radiother Oncol 2010;96(September (3)):308-10.

32. Boellaard R, Oyen WJG, Hoekstra CJ, et al. The Netherlands protocol for standardisation and quantification of FDG whole body PET studies in multi-centre trials. Eur J Nucl Med Mol Imaging 2008;35(December (12)):2320-33.

33. Boellaard R. Standards for PET image acquisition and quantitative data analysis. J Nucl Med 2009;50(May (Suppl. 1)):11S-20S.

34. Shankar LK, Hoffman JM, Bacharach S, et al. Consensus recommendations for the use of ${ }^{18}$ F-FDG PET as an indicator of therapeutic response in patients in National Cancer Institute Trials. J Nucl Med 2006;47(June (6)):1059-66.

35. Ling CC, Humm J, Larson S, et al. Towards multidimensional radiotherapy (MD-CRT): biological imaging and biological conformality. Int J Radiat Oncol Biol Phys 2000;47(June (3)):551-60.

36. Davis JB, Reiner B, Huser M, Burger C, Székely G, Ciernik IF. Assessment of ${ }^{18} \mathrm{~F}$ PET signals for automatic target volume definition in radiotherapy treatment planning. Radiother Oncol 2006;80(July (1)):43-50.

37. Bentzen SM. High-tech in radiation oncology: should there be a ceiling? Int J Radiat Oncol Biol Phys 2004;58(February (2)):320-30

38. Zaidi $\mathrm{H}, \mathrm{El}$ Naqa I. PET-guided delineation of radiation therapy treatment volumes: a survey of image segmentation techniques. Eur J Nucl Med Mol Imaging 2010;37(November (11)):2165-87.

39. Lee JA. Segmentation of positron emission tomography images: some recommendations for target delineation in radiation oncology. Radiother Oncol 2010;96(September (3)):302-7.

40. Erdi YE, Mawlawi O, Larson SM, et al. Segmentation of lung lesion volume by adaptive positron emission tomography image thresholding. Cancer 1997;80(December (12 Suppl.)):2505-9.

41. Daisne J-F, Sibomana M, Bol A, Doumont T, Lonneux M, Grégoire V. Tri-dimensional automatic segmentation of PET volumes based on measured source-to-background ratios: influence of reconstruction algorithms. Radiother Oncol 2003;69(December (3)):247-50.

42. Hoffmann AL, van Dalen JA, Lee J, et al. Assessment of (18)F PET signals for automatic target volume definition in radiotherapy treatment planning. Radiother Oncol 2007;83(April (1)):102-3, author reply 103.

43. King MA, Long DT, Brill AB. SPECT volume quantitation: influence of spatial resolution, source size and shape, and voxel size. Med Phys 1991;18(October (5)):1016-24.

44. Biehl KJ, Kong F-M, Dehdashti F, et al. ${ }^{18}$ F-FDG PET definition of gross tumor volume for radiotherapy of non-small cell lung cancer: is a single standardized uptake value threshold approach appropriate? J Nucl Med 2006;47(November (11)):1808-12.

45. Zaidi H. Organ volume estimation using SPECT. IEEE Trans Nuclear Sci 1996;43(June (3)):2174-82.

46. Yaremko B, Riauka T, Robinson D, et al. Thresholding in PET images of static and moving targets. Phys Med Biol 2005;50(December (24)):5969-82.

47. Geets X, Lee JA, Bol A, Lonneux M, Grégoire V. A gradient-based method for segmenting FDG-PET images: methodology and validation. Eur J Nucl Med Mol Imaging 2007;34(September (9)):1427-38.

48. Fogh S, Farach A, Intenzo C, et al. Pathologic correlation of PET-CT based auto contouring for radiation planning in lung cancer. Bodine J [Internet] 2010;3(December (1)). Available from: http://jdc.jefferson.edu/bodinejournal/vol3/iss1/30

49. Long DT, King MA, Sheehan J. Comparative evaluation of image segmentation methods for volume quantitation in SPECT. Med Phys 1992;19(April (2)):483-9.

50. Protocolo Español de Control de Calidad en Radiodiagnóstico (Aspectos técnicos) SEFM/SEPR; 2003.

51. Protocolo Español de Control de Calidad en Sistemas de Planificación de Terapia con Radiaciones Ionizantes. SEFM; 2002.

52. Control de Calidad de Equipos TC para Radioterapia. Guía Práctica. Del Grupo GOCO Revista Física Méd 2005;6(1): 37-45.

53. Mutic S, Palta JR, Butker EK, et al. Quality assurance for computed-tomography simulators and the computed-tomography-simulation process: report of the AAPM Radiation Therapy Committee Task Group No. 66. Med Phys 2003;30(October (10)):2762-92.

54. Quality Assurance for PET and PET/CT System. IAEA HUMAN HEALTH SERIES; 2009.

55. Protocolo Nacional de Control de Calidad en la Instrumentación en Medicina Nuclear. SEFM, SEMN, SEPR; 1999.

56. Shreve P, Townsend DW, editors. Clinical PET-CT in radiology (integrated imaging in oncology). Springer; 2008.

57. Hubbell, Seltzer SM. Tables of X-ray mass attenuation coefficients and mass energy-absorption coefficients $1 \mathrm{keV}$ to $20 \mathrm{meV}$ for elements $\mathrm{z}=1$ to 92 and 48 additional substances of dosimetric interest. Available at: http://www.nist.gov/pml/data/xraycoef/index.cfm [accessed 09.09.12].

58. Daisne J-F, Duprez T, Weynand B, et al. Tumor volume in pharyngolaryngeal squamous cell carcinoma: comparison at CT, MR imaging, and FDG PET and validation with surgical specimen. Radiology 2004;233(October (1)):93-100.

59. Riegel AC, Bucci MK, Mawlawi OR, et al. Target definition of moving lung tumors in positron emission tomography: correlation of optimal activity concentration thresholds with object size, motion extent, and source-to-background ratio. Med Phys 2010;37(April (4)):1742-52.

60. Faasse T, Shreve P. Patient and image data management in positron emission tomography-computed tomography for radiation therapy and therapy response assessment. Semin Ultrasound CT MR 2010;31(December (6)):480-9. 